\title{
MAGNETOELECTRIC EFFECT IN MAGNETIC MATERIALS
}

\author{
K. SIRATORI \\ Department of Physics, Faculty of Science, Osaka University \\ Toyonaka, Osaka, Japan \\ K. Konn \\ School of Science and Engineering, Waseda University \\ Shinjuku, Tokyo, Japan \\ AND E. KITA \\ Institute of Applied Physics, University of Tsukuba \\ Tsukuba, Ibaraki, Japan \\ (Received December 31, 1991)
}

\begin{abstract}
Magnetoelectric effect in magnetic insulators is reviewed. After an intuitive explanation of the effect in antiferromagnetic $\mathrm{Cr}_{2} \mathrm{O}_{3}$, the following topics are discussed on the experiments. Measurement and controlling of antiferromagnetic domains as well as antiferromagnetic domain wall motion. Expansion of the free energy of crystals in terms of magnetic and electric fields and polarizations. Information on the magnetic symmetry of crystals. Investigation of mechanisms of magnetoelectric effect. Excited states and optical observation.
\end{abstract}

PACS numbers: $75.80 .+q$

\section{Introduction}

In some insulating crystals, external electric field can induce magnetic moment and external magnetic field induces electric polarization. Such a phenomenon is called "magnetoelectric" effect.

This effect was proposed at first for molecules such as NO which has odd number of electrons, thus uncompensated spins, as well as electric dipole moment. After experiments on gases with negative results, Van Vleck explained the reason for the absence of the effect [1]. 
Essentially, the explanation was based on the fact that the symmetry of the two kinds of polarizations or fields, electric and magnetic, is different. Because the electric field and polarization are polar vectors, operation of spacial inversion $I$ reverses their direction. However, this operation has no effect on the magnetic moment or field since they are axial vectors. On the other hand, time inversion operation $R \equiv t \rightarrow-t$ reverses the magnetic moment or field without any effect on the electric polarization or field. Application of the electric field removes inversion symmetry of the system and results in the appearance of the electric polarization. Time reversal remains, however, as a symmetry operation and no magnetic moment can appear. Note that the gas, as a whole, has inversion and time-reversal symmetries, even though individual molecules do not have both. The above conclusion is based on this fact and restricted to the linear effect.

In the case of magnetic crystals, both symmetries can be absent. In such a case, linear magnetoelectric effect is possible. This point was clearly made by Landau and Lifshitz in their famous textbook [2] to which almost all the papers referred at the first stage of the investigation. The starting point on an actual crystal was marked by Dzyaloshinskii [3] by the prediction of the effect in the antiferromagnetic $\mathrm{Cr}_{2} \mathrm{O}_{3}$. Shortly after his prediction, Astrov [4,5] and Folen et al. [6] reported the experimental observation. By this paper of Dzyaloshinskii, together with the discovery of the antisymmetric exchange interaction and the explanation of the parasitic ferromagnetism of $\alpha-\mathrm{Fe}_{2} \mathrm{O}_{3}$ by that interaction [7], the phenomenological approach of the Landau school made an impressive appearance in the field of magnetism.

In these 30 years after the discovery, linear magnetoelectric effect has been observed in many magnetic compounds, including $\mathrm{Fe}_{3} \mathrm{O}_{4}$ and $\mathrm{YIG}$, yttrium iron garnet. Higher-order effects, the magnetic moment proportional to the square of the electric field strength or the magnetic susceptibilities dependent on the external electric field, were also discovered experimentally.

Magnetoelectric effect is an effect of the externally applied electric or magnetic field on the physical properties of crystals. The electronic state of the crystal is modified by the externally applied electric field and the modification is detected by the change of the net magnetic moment. The effect gives a unique method to get an insight into solids, though the possibility has not been opened up fully. It might be compared with the effect of compression on the physical properties of solids. One difference is that the energy of the compression in a crystal can be much larger than that of the electric field. Magnetoelectric effect is not so large in the magnitude. Another difference is that the observation of the magnetoelectric effect is restricted to insulators.

There is a symmetry between the effect of the electric and the magnetic field, as will be shown in Sec. 3. In the following, however, the effect of the electric field on the magnetic properties will be discussed mainly.

On the magnetoelectric effect, an international conference was held in 1973. The proceedings of the conference [8] as well as a book by O'Dell [9] will be beneficial to understand basic concepts and the data accumulated within about 10 years after the discovery of the effect. 


\section{An intuitive consideration}

\subsection{A case study of $\mathrm{Cr}_{2} \mathrm{O}_{3}$}

Let us start from $\mathrm{Cr}_{2} \mathrm{O}_{3}$, the starting material of the investigation. Figure 1 shows the corundum structure of this crystal. The point symmetry is rhombohedral $R \overline{3} c$, with a unit cell containing four crystallographically equivalent cations, octahedrally coordinated by six oxygens. This structure can be understood to be constructed by triangular lattices of anions and honeycomb lattice of cations, stacking along the [111] axis of the rhombohedral unit cell. The axis will be denoted

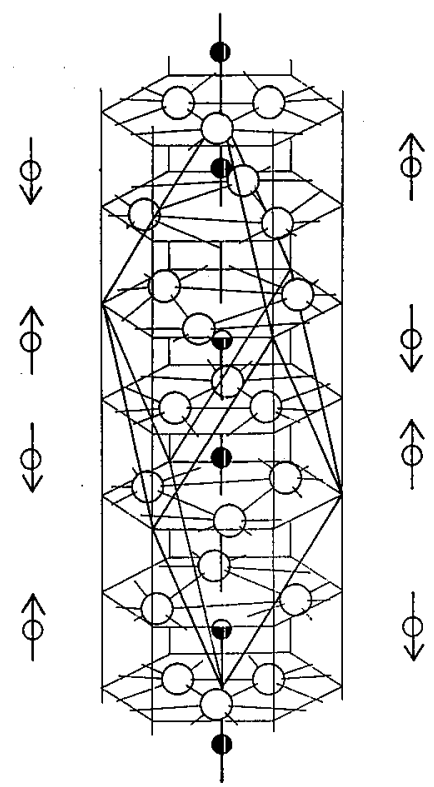

Fig. 1. Hexagonal (fine lines) and rhombohedral (solid lines) unit cell of the corundum structure. Large spheres are anions $\left(\mathrm{O}^{2-}\right)$ and small spheres are cations. Two equivalent antiferromagnetic structures in $\mathrm{Cr}_{2} \mathrm{O}_{3}$ are shown by arrows, at both sides of the figure.

$z$ or $c$ axis in the following, referred to the hexagonal frame. The coordination octahedron in this crystal is not regular but distorted. The upper triangle of oxygen atoms coordinating to the uppermost cation in the figure, for example, is smaller than the lower triangle, whereas the situation is reversed for the lowermost cation. We will call the former cation site as [ +$]$ and the latter site as [-]. Because of this distortion, the crystalline field at the cation has the $c$ component, + or - according to whether the site belongs to the $[+]$ or $[-]$ type. The $[+]$ site is converted to the [-] site by inversion $I$. We can assume that the electric polarization exists at each cation sites, directed inversely for $[+]$ and $[-]$ sites.

Below about $300 \mathrm{~K}, \mathrm{Cr}_{2} \mathrm{O}_{3}$ is an antiferromagnet with the spins parallel to the $c$ axis. The antiferromagnetic sublattices will be specifed by $\uparrow$ and $\downarrow$. As it 
is shown in the figure, the antiferromagnetic structure of $\mathrm{Cr}_{2} \mathrm{O}_{3}$ is characterized by the fact that the $\uparrow$ or $\downarrow$ lattice is identical with the $[+]$ or $[-]$ lattice. Thus $I R$, which changes $[+]$ to $[-]$ after reversing the spin direction, keeps the crystal unchanged. $I$ or $R$ alone is not the symmetry element of the crystal below the Néel point.

Now, let us apply the electric field along the $c$ axis. The field will shift all the cations, say, upward relative to the anions and the equivalence of the $[+]$ and the $[-]$ sublattices is removed. According to the above mentioned correspondence of sublattices, this means that the equivalence of the $\uparrow$ and $\downarrow$ sublattices is removed. Magnetic moments of these two antiferromagnetic sublattices need not cancel with each other and the net moment can appear along the $c$ axis. Note that $\mathrm{Cr}^{3+}$ spins lie along the $c$ axis. The electric field along the $c$ axis does not violate the threefold symmetry of the crystal along the axis and spins do not tilt from the axis.

When the electric field is applied within the $c$ plane, the equivalence of the two sublattices is not removed. However, the principal axis of the crystalline field of $\mathrm{Cr}^{3+}$ ions is tilted from the $c$ axis. When tilted, directions of the principal axes are not the same for $[+]$ and $[-]$ sublattices since the original component of the linear crystalline field along the $c$ axis is reversed in the two sublattices. The easy directions of the spins in the $\uparrow$ and $\downarrow$ sublattices are not parallel and net moment can appear within the $c$ plane, along the applied electric field. This is a parasitic ferromagnet of $\mathrm{NiF}_{2}$ type [10]. At the same time, it was pointed out that the antisymmetric Dzyaloshinskii interaction, $D \cdot\left[\boldsymbol{S}_{\uparrow} \times S_{\downarrow}\right]$, appears with the vector $D$ perpendicular to both applied electric field and the $c$ axis [11].

As a whole, the effect is expressed by the following equation:

$$
\begin{aligned}
& \boldsymbol{M}=\alpha \cdot \boldsymbol{E}, \\
& \alpha=\left(\begin{array}{ccc}
\alpha_{11} & 0 & 0 \\
0 & \alpha_{11} & 0 \\
0 & 0 & \alpha_{33}
\end{array}\right) .
\end{aligned}
$$

Equivalence of $\alpha_{11}$ and $\alpha_{22}$ is assured by the 3-fold symmetry along the $c$ axis of the crystal. An example of magnetoelectric susceptibilities is shown in Fig. 2 as a function of temperature [12]. If we use cgs-Gauss unit, magnetoelectric susceptibility is an innominal number. Naturally, the effect disappears at the Néel point because symmetry $R$ is recovered.

The above description shows the importance of the crystalline electric field along the $c$ axis, which distinguishes $[+]$ and $[-]$ sublattices. If there is no $c$-component of the crystalline field at the cation site, the equivalence of both sublattices is maintained under the external electric field along the $c$ axis. Electric field applied within the $c$ plane will not tilt the principal axis of the spin Hamiltonian. We cannot expect the appearance of the magnetic moment by the external electric field.

Existence of the crystalline electric field at the cation site is not sufficient for the effect. If $\uparrow(\downarrow)$ sublattice is different from $[+]([-])$ and contains equal number of both sites, the effect of the external electric field cancels out within each antiferromagnetic sublattice. This is the case of $\alpha-\mathrm{Fe}_{2} \mathrm{O}_{3}$. 


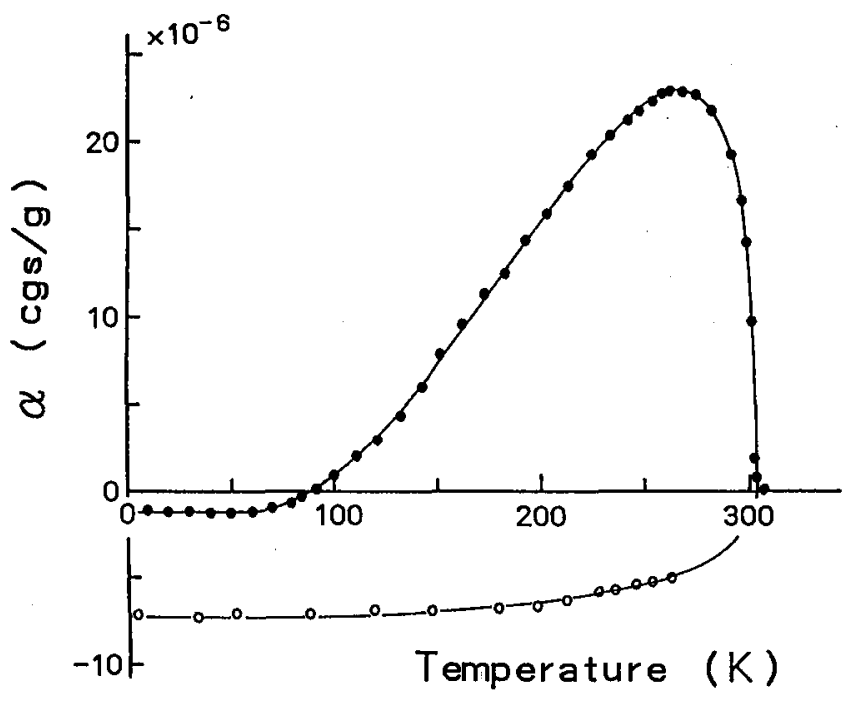

Fig. 2. Temperature dependence of the linear magnetoelectric coefficient, $\alpha_{11}$ (open circles) and $\alpha_{33}$ (solid circles), in $\mathrm{Cr}_{2} \mathrm{O}_{3}$ [12]. Measurement was carried out by the use of SQUID, after parallel magnetoelectric cooling along the $c$ axis.

Strictly, all crystals do not have inversion symmetry when the external electric field is applied. In the case where inversion is maintained originally, however, the amount of the breaking is proportional to the electric field when the field is not too large. We can expect that the coefficient of the above mentioned linear effect is proportional to the external field and the total effect is proportional to $E^{2}$. The same argument is valid for the external magnetic field. The effect proportional to $H^{2}$ exists even in the paramagnetic crystal.

\subsection{Antiferromagnetic domains}

In the above explanation of $\mathrm{Cr}_{2} \mathrm{O}_{3}$, it is evident that the direction of the net moment, which appears by the external electric field, is determined by the correspondence of the $[+]$ and the $[-]$ sublattices to the $\uparrow$ and $\downarrow$. Let us assume that the electric field along the $+c$ increases the thermal average of spins at the $[+]$ site. Then, the net moment due to the magnetoelectric effect points $+c$ if $[+]$ site composes $\uparrow$ sublattice whereas $-c$ if $[+]$ site is $\downarrow$. These two kinds of correspondence mean that magnetoelectric effect can distinguish two kinds of antiferromagnetic domains.

In contrast to the ferromagnetic domains which appear to minimize the free energy of the crystal, antiferromagnetic domains are constructed because they are degenerated in the energy. If antiferromagnetic ordering starts at one point and develops to the whole crystal, there will be only one domain. Ordinarily, however, that is not the case. Ordering starts from many points simultaneously, many domains appear and the resulting structure will remain stable. Except for 
the magnetoelectric crystals, there is no way to differentiate one antiferromagnetic domain from the other."

By the application of the electric field on $\mathrm{Cr}_{2} \mathrm{O}_{3}$, cooled below $T_{\mathrm{N}}$ without fields, we can expect that the directions of the induced magnetic moments are different from domain to domain. The antiferromagnetic domain structure will be visualized by observing polarity of the induced magnetic moment, by the use of, say, Kerr technique, though the necessitated sensitivity is very high. The Faraday effect may be easier from the sensitivity consideration, though the effect is integrated along the optical path. On the optical experiments; see Sec. 6.

The signal amplitude of the ordinary magnetoelectric effect is proportional to the net moment, thus to the unbalance of both domains. If they are the same, no signal is expected. If magnetic and electric fields are applied simultaneously, the energy of domains becomes different depending on whether the electrically induced magnetic moment is parallel or antiparallel to the magnetic field. This energy difference results in the change of the volume of each kind of domains through domain wall motion. The amount of the resulted volume unbalance can be measured by magnetoelectric effect. One example of such a measurement is shown in Fig. 3 [16], where non-reciprocal rotation of the optical polarization was detected (see Sec. 6). A hysteresis curve, similar to that in the magnetization process of a ferromagnet, was observed. In the case of Fig. 3, two kinds of domains are not equivalent and one is more stable than the other. Since antiferromagnetic domains appear in thermally inequilibrated state, such an inequivalence is not unexpected.

In the case where electrical conductivity is too high to maintain sufficient electric field stably within the specimen, the electric field pulses are eflective. This process is called magnetoelectric poling. Figure 4 shows an example for $\mathrm{Fe}_{3} \mathrm{O}_{4}$ at $77 \mathrm{~K}[17]$.

In order to measure correctly the magnitude of the intrinsic magnetoelectric susceptibility of an antiferromagnet, measurements should be carried out on a specimen in which antiferromagnetic domains are eliminated. They correspond to the state of saturation in Fig. 3. Cooling of the specimen through the transition point in the presence of external magnetic and electric field is effective for this purpose. This is named magnetoelectric cooling.

By measuring the time-derivative of the signal, kinetics of the antiferromagnetic domain wall motion can be investigated. Brown and O'Dell [18] applied a constant electric field $E$ along the $c$ axis of $\mathrm{Cr}_{2} \mathrm{O}_{3}$, which is placed in a parallel magnetic field $H$. Electric pulses are superposed along the axis to measure

*In the case of $\mathrm{NiO}$ with $\mathrm{NaCl}$ structure, e.g., spins in one of the cubic $\{111\}$ plane order ferromagnetically and the planes stack antiferromagnetically [13]. Easy direction of spins is one of the $\langle 11 \overline{2}\rangle$ axes within the plane $[14,15]$. Because there are four $\{111\}$ planes and three $\langle 11 \overline{2}\rangle$ axes in one (111) plane, there are twelve kinds of domains with different antiferromagnetic stacking and spin direction. Such domains can be detected and controlled by external stress and magnetic field, since the crystal lattice is distorted by exchange and magnetostriction and the magnetic susceptibility is anisotropic. The antiferromagnetic domains discussed above are of the different kind. In the crystal where antiferromagnetic structure as well as spin axis are determined, still there remains a degeneracy because one spin can direct either up or down. That is the point of the above argument. Rhombohedral $\mathrm{Cr}_{2} \mathrm{O}_{3}$ has only this degeneracy. 


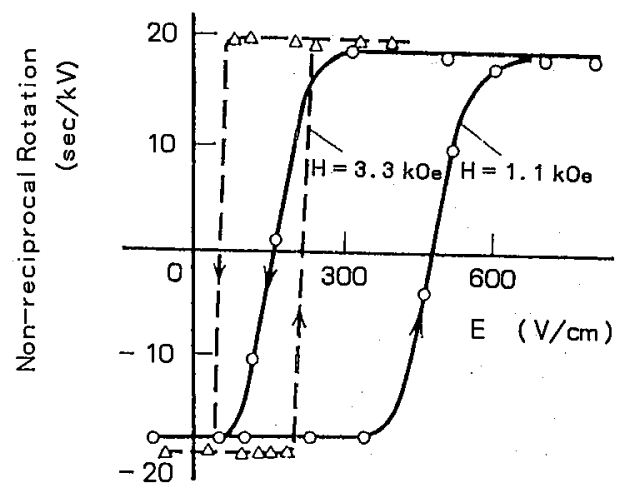

Fig. 3. Antiferromagnetic hysteresis curves in $\mathrm{Cr}_{2} \mathrm{O}_{3}$, shifted to one direction. Non-reciprocal optical rotation due to the application of the electric field was measured as a function of electric field, in a constant magnetic field. On the effect, see Sec. 6 . Reproduced with permission from Ref. [16]. Copyright by the Academy of Sciences of USSR.

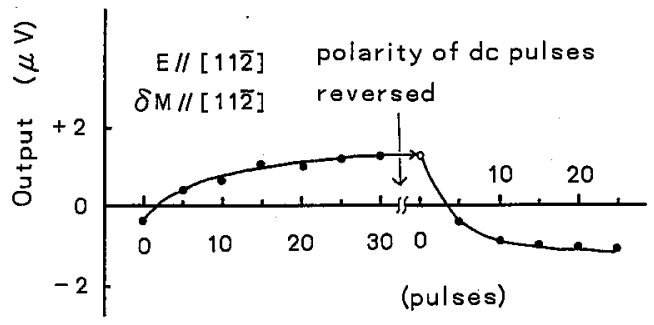

Fig. 4. Effect of the magnetoelectric poling at $77 \mathrm{~K}$ for $\mathrm{Fe}_{3} \mathrm{O}_{4}$. The output of the linear magnetoelectric effect is plotted against the number of dc electric pulses, $2.5 \mathrm{kV} / \mathrm{cm}$ in heights and $0.7 \mathrm{~ms}$ duration, applied before the measurement. Reproduced with permission from Ref. [17]. Copyright by the Physical Society of Japan.

magnetoelectric susceptibility as a function of time (as shown in Fig. 5). Switching time $t_{\mathrm{s}}$ was a function of the product $E H$ and could be expressed as

$$
1 / t_{\mathrm{s}}=C\left\{E H-(E H)_{\mathrm{thr}}\right\} \text {. }
$$

By annealing of the specimen, the constant factor $C$ increases whereas the threshold value $(E H)_{\text {thr }}$ decreases. In a strained crystal, e.g., as-grown crystal by Verneuil method, $t_{\mathrm{s}}$ approaches a constant value with an increase in $E H$. These behaviors are similar to the wall switching in ferrites with rectangular hysteresis. Brown and O'Dell have estimated the width of the domain wall in $\mathrm{Cr}_{2} \mathrm{O}_{3}$ as $200-1000 \AA$, from the wall velocity.

In a special case of a screw spin structure, antiferromagnetic domains, clockwise and anticlockwise rotation of spins for fixed screw and spin axes, can be detected by polarized neutron diffraction [19, 20]. This degeneracy can be 

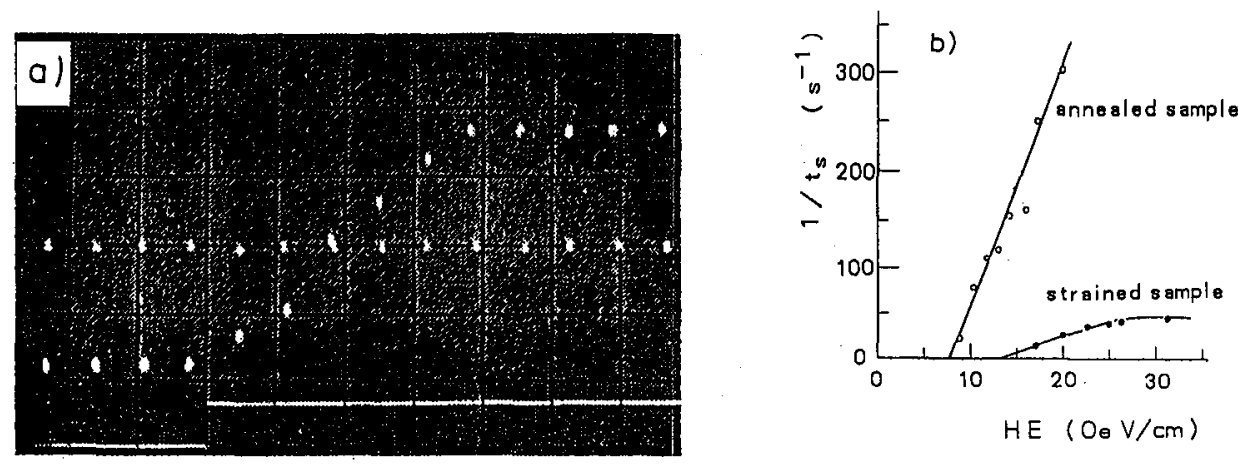

Fig. 5. (a) Switching of the antiferromagnetic domains in $\mathrm{Cr}_{2} \mathrm{O}_{3}$. The dots indicate magnetoelectric susceptibility, with reference points at the center. A smooth line at the bottom indicates applied dc electric field of $6.5 \mathrm{kV} / \mathrm{cm}$. Static magnetic field of $3.4 \mathrm{kOe}$ was applied along the $c$ axis, parallel to the electric field. Scale of the abscissa is 10 $\mathrm{ms} /$ division. (b) Dependence of the switching velocity at $297 \mathrm{~K}$ on the product $E H$, for annealed and as-grown specimens. Reproduced with permission from Ref. [18]. (C)1969 IEEE).
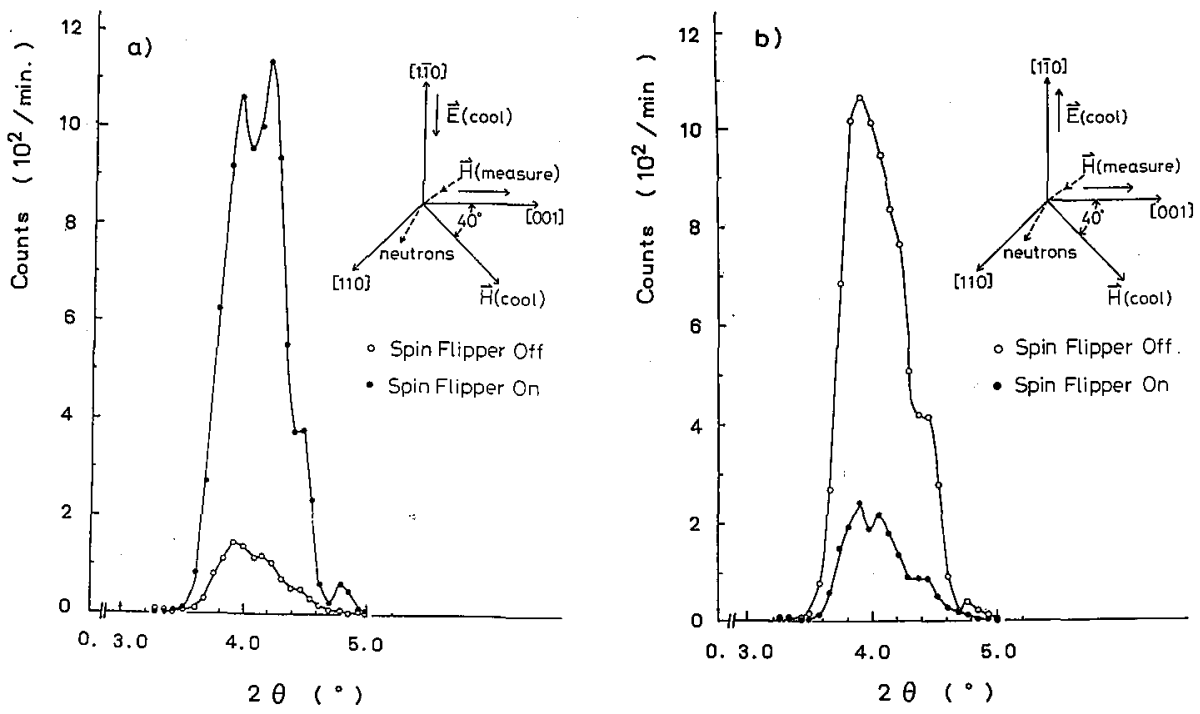

Fig. 6. Dependence of neutron intensity on the polarization direction of incident neutrons at the satellite $\left(0,0, q_{0}\right)$ of $\mathrm{ZnCr}_{2} \mathrm{Se}_{4}$ with screw spin structure, after magnetoelectric cooling as shown in the figure. Cooling electric field was $2.5 \mathrm{kV} / \mathrm{cm}$, cooling magnetic field was $12 \mathrm{kOe}$, measuring magnetic field was $3.4 \mathrm{kOe}$ and neutron wavelength was $1.57 \AA$. The sense of the screw structure is controlled by the direction of cooling fields, as was detected by the diffraction intensity depending on the polarity (spin direction) of incident neutrons. Reproduced with permission from Ref. [21]. Copyright by the Physical Society of Japan. 


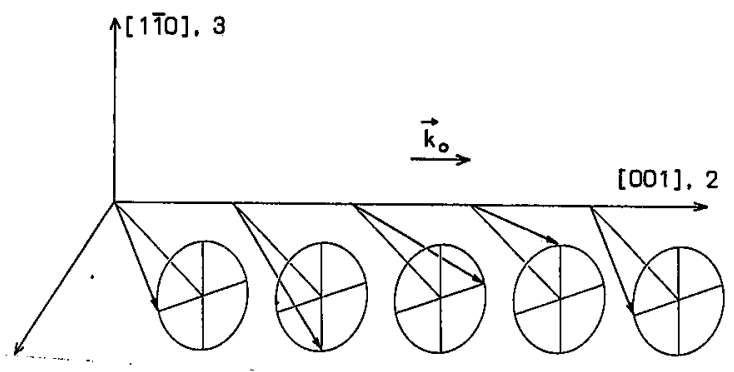

Fig. 7. Conical spin structure and the Cartesian coordinate. Reproduced with permission from Ref. [21]. Copyright by the Physical Society of Japan.

eliminated by magnetoelectric cooling. Figure 6 shows an example for $\mathrm{ZnCr}_{2} \mathrm{Se}_{4}$ [21]. This crystal has a cubic normal spinel structure and spins of $\mathrm{Cr}^{3+}$ ions order in a proper screw structure along one of the $\langle 100\rangle$ axes below $20 \mathrm{~K}$. Because of the anisotropy of the magnetic susceptibility in the screw structure, external magnetic field can fix the screw axis along one of the $\langle 100\rangle$. No first-order magnetoelectric effect can be expected in the proper screw spin structure, since it has $R$ (+ translation by one half of the screw pitch along the screw axis) symmetry. However, $R$ is eliminated in the case of the conical spin structure, with constant spin components along the spin axis (Fig. 7). By the insert in Fig. 6, the component of the cooling magnetic field along the [001] axis is for the determination of the screw axis and that along the [110] is for the control of the sense of the screw structure.

\section{Free energy of the crystal}

In order to describe the argument in the last section more rigorously, we will expand free energy of the crystal in the power series, in terms of the external magnetic field $\boldsymbol{H}$ and the electric field $\boldsymbol{E}$ :

$$
F=F_{0}-\boldsymbol{P}_{0} \cdot \boldsymbol{E}-\boldsymbol{M}_{0} \cdot \boldsymbol{H}-\boldsymbol{E} \cdot \frac{\chi_{\mathrm{e}}}{2} \cdot \boldsymbol{E}-\boldsymbol{H} \cdot \frac{\chi}{2} \cdot \boldsymbol{H}-\boldsymbol{E} \cdot \alpha \cdot \boldsymbol{H}-\ldots
$$

$F_{0}$ is the free energy independent of $\boldsymbol{E}$ or $\boldsymbol{H}$. Derivative of $\boldsymbol{F}$ in $\boldsymbol{H}$ gives magnetic moment and in $\boldsymbol{E}$ electric polarization

$$
\begin{gathered}
\boldsymbol{M}=-\frac{\partial F}{\partial \boldsymbol{H}}=\boldsymbol{M}_{0}+\chi \cdot \boldsymbol{H}+\alpha \cdot \boldsymbol{E}, \\
\boldsymbol{P}=-\frac{\partial F}{\partial \boldsymbol{E}}=\boldsymbol{P}_{0}+\chi_{\mathrm{e}} \cdot \boldsymbol{E}+\alpha \cdot \boldsymbol{H} .
\end{gathered}
$$

The first-order magnetoelectric effect is due to the sixth term of Eq. (4). If operation $S$ is a symmetry element of the crystal, Eq. (4) is an invariant to $S$ :

$$
S(F)=F \text {. }
$$

The magnetic symmetry of the crystal, which is reflected in operations $S$ in Eq. (7), determines the coefficients in Eqs. (4)-(6), $\alpha$, etc. Conversely, the magnetic point symmetry of the crystal can be determined by the experimentally verified form 
of the coefficient tensor $\alpha$ in Eq. (4). This is one of the main objectives of the investigation of magnetoelectric effect. In Table I, all the magnetic symmetries which allow linear magnetoelectric effect are tabulated with the magnetoelectric susceptibility tensors. (For the magnetic point group, see, e.g., [27].) Examples of the material are also shown.

Equations (4)-(6) show that the coefficient of the electric polarization against the external magnetic field is the transposed tensor of the coefficient of the magnetic moment against electric field. In several cases, this relation was examined experimentally, though the absolute magnitude is rather difficult to be compared. See Fig. 8 [28].

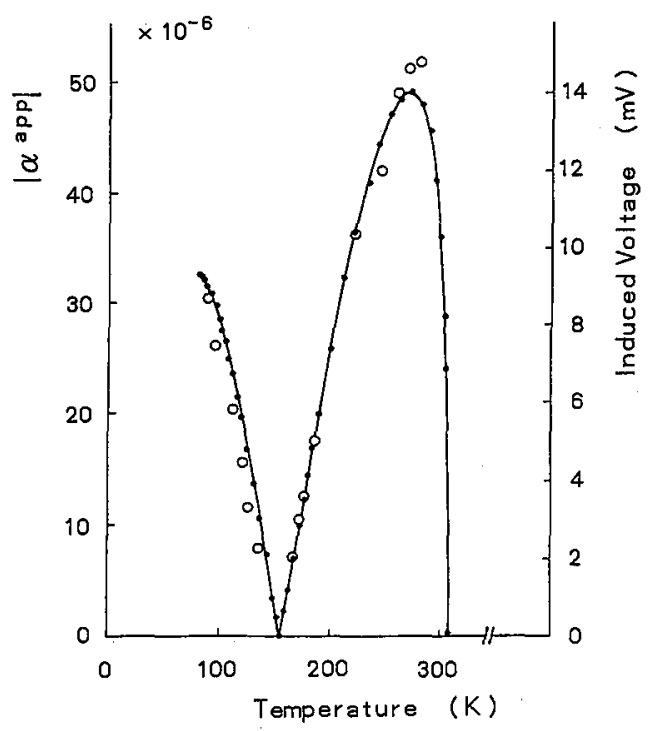

Fig. 8. Comparison of the temperature dependence of the magnetoelectric susceptibilities of $\mathrm{Cr}_{2} \mathrm{O}_{3}$ measured by applying external electric field (solid circles) and magnetic field (open circles). Both fields were parallel, about $30^{\circ}$ tilted from the $c$ axis. Reproduced with permission from Ref. [28]. Copyright by the American Physical Society.

The series expansion of the free energy in terms of $\boldsymbol{H}$ and $\boldsymbol{E}$ will continue as

$$
F=\ldots-\sum_{i, j, k}\left[\beta_{i j k} H_{i} E_{j} E_{k}+\gamma_{i j k} H_{i} H_{j} E_{k}\right]-\sum_{i, j, k, l} \delta_{i j k l} H_{i} H_{j} E_{k} E_{l}-\ldots
$$

The physical meaning of these terms have been described already in the last section. It is to be noted that the biquadratic term $\delta_{i j k l} H_{i} H_{j} E_{k} E_{l}$ has finite contribution in any crystals, although it is usually small.

The cause of magnetoelectric effects is not externally applied electric field itself but the electric polarization, displacement of atoms and deformation of electron clouds, induced by the field. Therefore, the expansion of the free energy in terms of $\boldsymbol{M}$ and $\boldsymbol{P}$, instead of $\boldsymbol{H}$ and $\boldsymbol{E}$, is more intuitive to understand the mechanisms of magnetoelectric effects. According to the general theory of Landau and 
TABLE I Magnetic point groups compatible with linear magnetoelectric effect. The forms of the magnetoelectric tensor are shown with some examples.

\begin{tabular}{|c|c|c|c|c|c|}
\hline Magnetic symmetry & \multicolumn{3}{|c|}{ ME-tensor } & Example & Ref. \\
\hline $1, \overline{1}^{\prime}$ & $\begin{array}{l}\alpha_{11} \\
\alpha_{21} \\
\alpha_{31} \\
\end{array}$ & $\begin{array}{l}\alpha_{12} \\
\alpha_{22} \\
\alpha_{32} \\
\end{array}$ & $\begin{array}{l}\alpha_{13} \\
\alpha_{23} \\
\alpha_{33} \\
\end{array}$ & $\begin{array}{l}\mathrm{Fe}_{3} \mathrm{O}_{4} \\
(1)\end{array}$ & {$[17]$} \\
\hline $2, m^{\prime}, 2 / m^{\prime}$ & $\begin{array}{c}\alpha_{11} \\
\alpha_{21} \\
0\end{array}$ & $\begin{array}{c}\alpha_{12} \\
\alpha_{22} \\
0 \\
\end{array}$ & $\begin{array}{c}0 \\
0 \\
\alpha_{33} \\
\end{array}$ & $\begin{array}{l}\mathrm{Ni}-\mathrm{I} \\
\text { Boracite } \\
\left(m^{\prime}\right)\end{array}$ & {$[22,65]$} \\
\hline $2^{\prime}, m, 2^{\prime} / m$ & $\begin{array}{r}0 \\
0 \\
\alpha_{31} \\
\end{array}$ & $\begin{array}{c}0 \\
0 \\
\alpha_{32} \\
\end{array}$ & $\begin{array}{c}\alpha_{13} \\
\alpha_{23} \\
0 \\
\end{array}$ & $\begin{array}{l}\mathrm{ErOOH} \\
\left(2^{\prime} / m\right)\end{array}$ & {$[23]$} \\
\hline $222, m^{\prime} m^{\prime} 2, m^{\prime} m^{\prime} m^{\prime}$ & $\begin{array}{c}\alpha_{11} \\
0 \\
0 \\
\end{array}$ & $\begin{array}{c}0 \\
\alpha_{22} \\
0 \\
\end{array}$ & $\begin{array}{c}0 \\
0 \\
\alpha_{33} \\
\end{array}$ & $\begin{array}{l}\mathrm{Cu}-\mathrm{Cl} \\
\text { Boracite } \\
\left(m^{\prime} m^{\prime} 2\right)\end{array}$ & {$[24]$} \\
\hline $22^{\prime} 2^{\prime}, m m 2, m^{\prime} m 2^{\prime}, m^{\prime} m m$ & $\begin{array}{l}0 \\
0 \\
0 \\
\end{array}$ & $\begin{array}{c}0 \\
0 \\
\alpha_{32} \\
\end{array}$ & $\begin{array}{c}0 \\
\alpha_{23} \\
0 \\
\end{array}$ & $\begin{array}{l}\mathrm{Ni}-\mathrm{Cl} \\
\text { Boracite } \\
\left(m^{\prime} m 2^{\prime}\right) \\
\end{array}$ & [25] \\
\hline $4, \overline{4}^{\prime}, 4 / m^{\prime}, 3, \overline{3}^{\prime}, 6, \overline{6}, 6 / m^{\prime}$ & $\begin{array}{c}\alpha_{11} \\
-\alpha_{12} \\
0 \\
\end{array}$ & $\begin{array}{c}\alpha_{12} \\
\alpha_{11} \\
0\end{array}$ & $\begin{array}{c}0 \\
0 \\
\alpha_{33} \\
\end{array}$ & & \\
\hline $4^{\prime}, \overline{4}, 4^{\prime} / m^{\prime}$ & $\begin{array}{c}\alpha_{11} \\
\alpha_{12} \\
0 \\
\end{array}$ & $\begin{array}{c}\alpha_{12} \\
-\alpha_{11} \\
0 \\
\end{array}$ & $\begin{array}{l}0 \\
0 \\
0 \\
\end{array}$ & & \\
\hline $\begin{array}{l}422,4 m^{\prime} m^{\prime}, \overline{4}^{\prime} 2 m^{\prime}, 4 m^{\prime} m^{\prime} m^{\prime} \\
32,3 m^{\prime}, \overline{3}^{\prime} m^{\prime}, 622,6 m^{\prime} m^{\prime} \\
\overline{6}^{\prime} m^{\prime} 2,6 / m^{\prime} m^{\prime} m^{\prime}\end{array}$ & $\begin{array}{c}\alpha_{11} \\
0 \\
0 \\
\end{array}$ & $\begin{array}{c}0 \\
\alpha_{11} \\
0 \\
\end{array}$ & $\begin{array}{c}0 \\
0 \\
\alpha_{33} \\
\end{array}$ & $\begin{array}{l}\mathrm{Cr}_{2} \mathrm{O}_{3} \\
\left(\overline{3}^{\prime} m^{\prime}\right)\end{array}$ & [5] \\
\hline $\begin{array}{l}4^{\prime} 22,4 m m^{\prime}, \overline{4} 2 m, \overline{4} 2^{\prime} m^{\prime} \\
4^{\prime} / m^{\prime} m m^{\prime}\end{array}$ & $\begin{array}{c}\alpha_{11} \\
0 \\
0\end{array}$ & $\begin{array}{c}0 \\
-\alpha_{11} \\
0 \\
\end{array}$ & $\begin{array}{r}0 \\
0 \\
0 \\
\end{array}$ & $\begin{array}{l}\mathrm{DyPO}_{4} \\
\left(4^{\prime} / m^{\prime} m m^{\prime}\right)\end{array}$ & [26] \\
\hline $\begin{array}{l}42^{\prime} 2^{\prime}, 4 m m, \overline{4}^{\prime} 2^{\prime} m, 4 / m^{\prime} m m \\
32^{\prime}, 3 m, \overline{3}^{\prime} m, 62^{\prime} 2,6 m m \\
\overline{6}^{\prime} m 2^{\prime}, 6 / m^{\prime} m m\end{array}$ & $\begin{array}{c}0 \\
-\alpha_{12} \\
0 \\
\end{array}$ & $\begin{array}{c}\alpha_{12} \\
0 \\
0 \\
\end{array}$ & $\begin{array}{l}0 \\
0 \\
0\end{array}$ & & \\
\hline $2 m, m^{\prime} 3,432, \overline{4}^{\prime} 3 m^{\prime}, m^{\prime} 3 m$ & $\begin{array}{c}\alpha_{11} \\
0 \\
0\end{array}$ & $\begin{array}{c}0 \\
\alpha_{11} \\
0\end{array}$ & $\begin{array}{c}0 \\
0 \\
\alpha_{11}\end{array}$ & & \\
\hline
\end{tabular}


Lifshitz, the free energy can be expanded by quadratic terms of $M$ and $P$ [29]. Of the terms in the series, the biquadratic term is important in the discussion here.

$$
F(M, P)=\ldots-\sum \Delta_{i j k l} M_{i} M_{j} P_{k} P_{l}-\ldots
$$

The variable in Eq. (9) is changed from $\boldsymbol{M}$ to $\boldsymbol{H}$ by Legendre transformation. In the case of ferromagnets where magnetization is saturated technically, we can assume

$$
\boldsymbol{M}=\boldsymbol{M}_{0}+\chi \boldsymbol{H} \text {. }
$$

If the crystal is pyroelectric at the same time, a similar transformation will be applied to $P$ :

$$
\boldsymbol{P}=\boldsymbol{P}_{0}+\chi_{\mathrm{e}} \boldsymbol{E} \text {. }
$$

Then

$$
\begin{aligned}
F(\boldsymbol{H}, \boldsymbol{E})= & \boldsymbol{F}(\boldsymbol{M}, \boldsymbol{P})-\boldsymbol{M} \cdot \boldsymbol{H}-\boldsymbol{P} \cdot \boldsymbol{E} \\
= & \ldots-\sum \Delta_{i j k l}\left(M_{0 i}+\chi H_{i}\right) \\
& \times\left(M_{0 j}+\chi H_{j}\right)\left(P_{0 k}+\chi_{\mathrm{e}} E_{k}\right)\left(P_{0 l}+\chi_{\mathrm{e}} E_{l}\right)-\ldots \\
= & \ldots-\sum \Delta_{i j k l}\left[M_{0 i} M_{0 j} P_{0 k} P_{0 l}\right. \\
& +\chi\left(M_{0 i} H_{j}+M_{0 j} H_{i}\right) P_{0 k} P_{0 l}+\chi_{\mathrm{e}} M_{0 i} M_{0 j}\left(P_{0 k} E_{l}+P_{0 l} E_{k}\right) \\
& +\chi^{2} P_{0 k} P_{0 l} H_{i} H_{j}+\chi_{\mathrm{e}}^{2} M_{0 i} M_{0 j} E_{k} E_{l} \\
& +\chi \chi_{\mathrm{e}}\left(M_{0 i} H_{j}+M_{0 j} H_{i}\right)\left(P_{0 k} E_{l}+P_{0 l} E_{k}\right) \\
& +\chi^{2} \chi_{\mathrm{e}} H_{i} H_{j}\left(P_{0 k} E_{l}+P_{0 l} E_{k}\right)+\chi \chi_{\mathrm{e}}^{2}\left(M_{0 i} H_{j}+M_{0 j} H_{i}\right) E_{k} E_{l} \\
& \left.+\chi^{2} \chi_{\mathrm{e}}^{2} H_{i} H_{j} E_{k} E_{l}\right]-\ldots
\end{aligned}
$$

Here, $\chi$ in Eq. (10) and $\chi_{\mathrm{e}}$ in Eq. (11) are assumed isotropic for simplicity. The term proportional to $E H$, as well as the terms to $E^{2} H$ and $E H^{2}$, comes about. Note that the coupling term of $M^{2} P^{2}$ in Eq. (9) affects the magnitude of the magnetic and electric polarization, $M_{0}$ and $P_{0}$, and susceptibilities $\chi$ and $\chi_{\mathrm{e}}$ through the terms $M_{0} \chi H P_{0}^{2}, M_{0}^{2} \chi_{\mathrm{e}} P_{0} E, \chi^{2} P_{0}^{2} H^{2}$ and $\chi_{\mathrm{e}}^{2} M_{0}^{2} E^{2}$, respectively.

In order to apply the analysis to ordered magnets in general, including helimagnets, the Fourier transformed form of Eq. (9) is convenient because such a structure possesses a certain periodicity, sometimes incommensurate to the atomic lattice. If the number of magnetic atoms is plural in the atomic primitive cell, we must distinguish modes or branches for the same wave number. In the following, we will denote mode and periodicity by a single parameter $q$. Then, the free energy is expressed as

$$
\begin{aligned}
& F(M, P)=\ldots-\sum \sum \tilde{\Delta}_{i j k l} M_{i}\left(q_{1}\right) M_{j}\left(q_{2}\right) P_{k}\left(q_{3}\right) P_{l}\left(q_{4}\right) \\
& \times \delta\left(q_{1}+q_{2}+q_{3}+q_{4}\right)-\ldots
\end{aligned}
$$

$\delta$-function means that the sum of the wave vectors in each branch should be zero.

Let us take the case of an ordered magnetic structure specified by finite $q_{0}$ where all $\boldsymbol{M}(q)$ vanishes except for $q=q_{0}$. In Eq. (10), the first term, $\boldsymbol{M}_{0}(q=0)$, vanishes. After the Legendre transformation,

$$
F(\boldsymbol{H}, \boldsymbol{E})=\ldots-\sum \tilde{\tilde{\Delta}}_{i j k l} \chi \chi_{\mathrm{e}} M_{0 i}\left(\boldsymbol{q}_{0}\right) H_{j} P_{0 k}\left(-q_{0}\right) E_{l}-\ldots
$$


Note that we apply homonegeous electric field with $q=0$ and detect magnetization with $q=0$, in the observation of magnetoelectric effect. Equation (14) means that the magnetic and electric structures should have the same mode and periodicity for magnetoelectric effect to exist.

The above condition is fulfiled in $\mathrm{Cr}_{2} \mathrm{O}_{3}$ where antiferromagnetic structure is the same as the structure of the (local) polarization, as mentioned in the last section. There appears a term proportional to $M_{0} P_{0} \chi \chi_{\mathrm{e}} E H$ in Eq. (14). No higher-order terms proportional to $E^{2} H$ or $E H^{2}$ can be expected. In the case of $\mathrm{ZnCr}_{2} \mathrm{Se}_{4}$ with screw spin structure, Eq. (14) indicates that the electric polarization with the wave vector $q_{0}$ appears below $T_{\mathrm{N}}$.
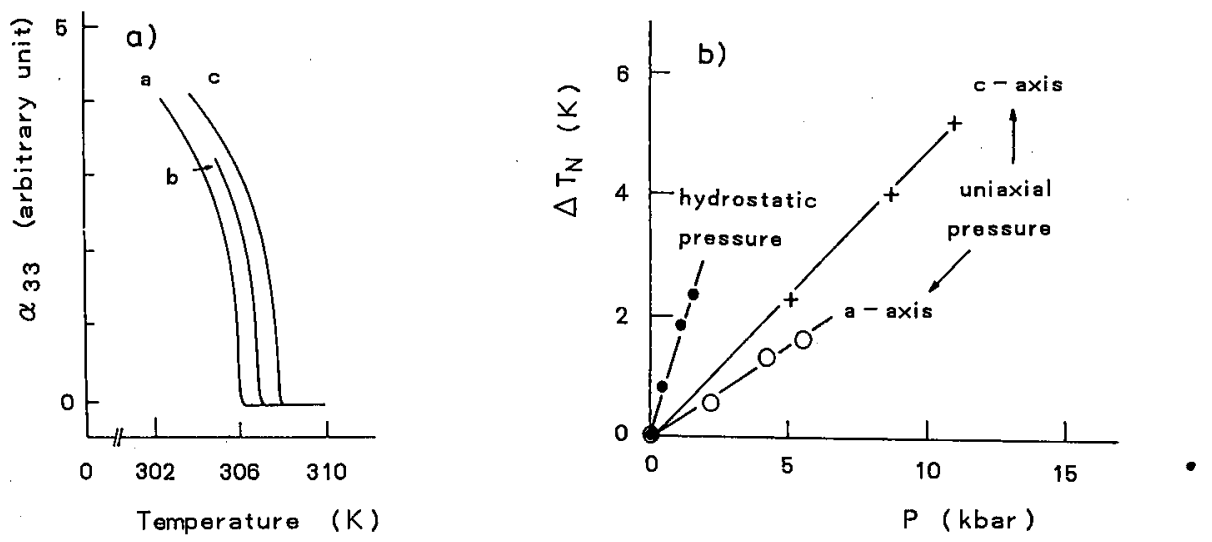

Fig. 9. (a) Determination of the Néel point of $\mathrm{Cr}_{2} \mathrm{O}_{3}$ under hydrostatic pressure. a: $0.07 \mathrm{kbar}$, b: $0.6 \mathrm{kbar}, \mathrm{c:} 1.2 \mathrm{kbar}$. (b) Dependence of the Néel point of $\mathrm{Cr}_{2} \mathrm{O}_{3}$ on the hydrostatic or uniaxial pressure along the $a$ or the $c$ axis. Reproduced with permission from Ref. [30]. Copyright by the American Physical Society.

It is to be noted that the coefficient of the term $E H$ is proportional to $M_{0}\left(q_{0}\right) \chi \cdot P_{0}\left(q_{0}\right) \chi_{\mathrm{e}}$ in any cases. For an antiferromagnet, measurement of magnetoelectric susceptibility at around the Néel point gives a method to determine the critical temperature as well as the temperature dependence of the sublattice magnetization $M_{0}\left(q_{0}\right)$, provided that other quantities can be assumed constant. This method is much easier and more precise than others, e.g., neutron diffraction, to determine $T_{\mathrm{N}}$ and $M_{0}$. Figure 9 shows examples of $T_{\mathrm{N}}$ determination for $\mathrm{Cr}_{2} \mathrm{O}_{3}$ under hydrostatic or uniaxial pressure* [30]. Critical index for the sublattice magnetization, $\beta$, has been determined for several substances and tabulated in Table II [31]. In Fig. 10 there are examples of $\log -\log$ plot for the determination of $\beta$.

The magnetization in the above arguments, except Zeeman term, should be read as the magnetic order parameter or the thermal average of electronic angular momenta, strictly. Since the observed magnetization is a product of that thermal

${ }^{*}$ In Fig. 9, the coefficient $\mathrm{d} T_{\mathrm{N}} / \mathrm{d} p$ for hydrostatic pressure is much larger than those for uniaxial compression both along the $a$ and $c$ axes. This fact suggests that the lattice strain under the uniaxial compression was not uniaxial. 


\section{TABLE II}

Neel point and the critical index $\beta$ for several oxide antiferromagnets, determined by the magnetoelectric effect [11].

\begin{tabular}{l|l|l|l}
\hline \hline Material & Néel point & \multicolumn{1}{|c|}{$\beta$} & Ref. \\
\hline $\mathrm{GdAlO}_{3}$ & $3.88 \mathrm{~K}$ & $0.31 \pm 0.01$ & {$[31]$} \\
$\mathrm{TbAlO}_{3}$ & 3.90 & 0.32 & {$[32]$} \\
$\mathrm{DyAlO}_{3}$ & 3.53 & $0.311 \pm 0.005$ & {$[33]$} \\
$\mathrm{GdVO}_{4}$ & 2.43 & $0.50 \pm 0.05$ & {$[34]$} \\
$\mathrm{DyPO}_{4}$ & 3.39 & 0.314 & {$[35]$} \\
$\mathrm{HoPO}_{4}$ & 1.39 & $0.315 \pm 0.01$ & {$[36]$} \\
$\mathrm{Cr}_{2} \mathrm{O}_{3}$ & 306 & $0.35 \pm 0.01$ & {$[37]$}
\end{tabular}

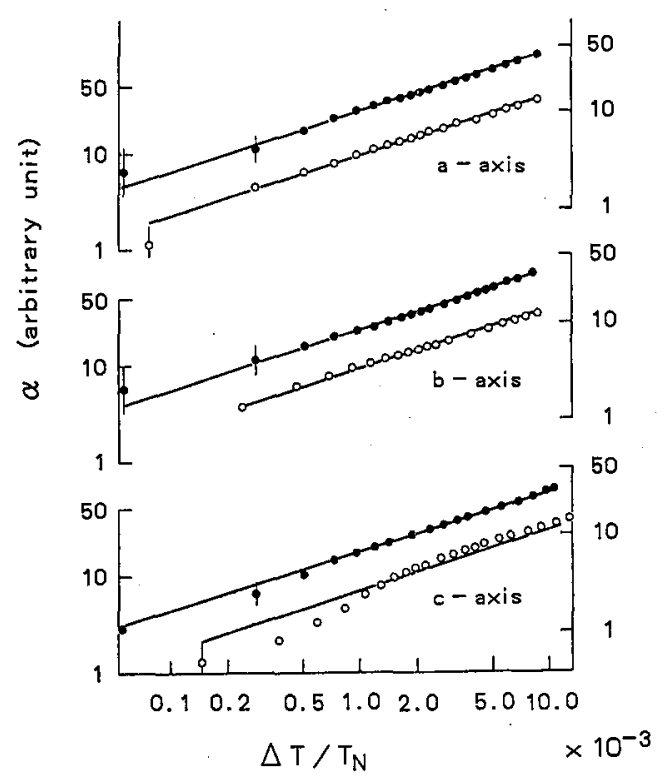

Fig. 10. Determination of the critical index $\beta$ for antiferromagnetic $\mathrm{GdAlO}_{3}$. Reproduced with permission from Ref. [31]. Copyright by the American Physical Society.

average and the $g$-factor

$$
\boldsymbol{M}=g\langle\boldsymbol{J}\rangle,
$$

the magnetoelectric effect can also arise through the electric polarization dependence of the $g$-factor. The magnetoelectric susceptibility $\alpha$ is not proportional to $M_{0} \chi$ in this case, but to $M_{0}$. This difference of the proportional factor gives a possibility to distinguish atomic mechanisms of the effect (see Sec. 5). 
So far, we have considered homogeneous systems. That is not the necessary condition, however, provided that the sensitivity of the measurement allows the detection. One example is the transition metal impurities in diamagnetic substances, observed by the electric field effect on the paramagnetic resonance [ 38 , 39]. This field of investigation was started also in 1961 on the deep levels in Si $\left(\mathrm{Fe}^{0}, \mathrm{Fe}^{+}\right)[40]$ and $\mathrm{Cr}^{3+}$ in ruby. Experimental data on ruby were utilized for the discussion of the mechanism of the magnetoelectric effect in $\mathrm{Cr}_{2} \mathrm{O}_{3}$ with the same crystal structure $[11,41]$. The electronic origin was discussed of the electric field dependence of the spin Hamiltonian coefficients [42].

Another possibility is lattice defects in an ordered magnet, though it has not been confirmed yet. This point will be described in the next section.

In the following sections, examples will be shown of the information obtainable through experiments of magnetoelectric effect.

\section{Determination of the magnetic symmetry of crystals}

As was mentioned in the last section and shown in Table I, the form of the linear magnetoelectric susceptibility tensor $\alpha$ gives information on the magnetic symmetry of the crystal. This is one of the main applications of magnetoelectric experiments. We will show two examples below. One is a large family of boracites and the other is YIG, yttrium iron garnet.

\subsection{Boracites}

Boracites are a family of halogenoborates with the general formula $\mathrm{M}_{3} \mathrm{~B}_{7} \mathrm{O}_{13} \mathrm{X}$. Usually $\mathrm{M}$ is a divalent metal ion such as iron group transition metals from $\mathrm{Cr}$ to $\mathrm{Zn}$ or $\mathrm{Mg}$ and $\mathrm{X}$ is a halogen ion, $\mathrm{F}^{-}$to $\mathrm{I}^{-}$, or $\mathrm{OH}^{-}$. Monovalent $\mathrm{Li}^{+}$ or divalent $\mathrm{S}^{2-}$ can also form the crystal with anion vacancies. For the sake of brevity, we will use a notation as $\mathrm{Cr}-\mathrm{Cl}$ boracite for the case of $\mathrm{M}=\mathrm{Cr}$ and $\mathrm{X}=$ $\mathrm{Cl}$.

The crystal of boracite is cubic and has a complicated structure composed of $\mathrm{BO}_{4}$ tetrahedra and $\mathrm{BO}_{3}$ triangles sharing their corners. The metal ion $\mathrm{M}$ is located at the center of an $\mathrm{MO}_{4} \mathrm{X}_{2}$ octahedron, which is sharing the apical $\mathrm{X}$ ions with its neighbor, forming $\mathrm{MO}_{2} \mathrm{X}$ network (Fig. 11). Since the 1960's, this family of compounds attracts the interests of solid state physicists, though the mineral boracite, $\mathrm{Mg}-\mathrm{Cl}$ boracite, has been known for a long time. Schmid, Ascher and their collaborators synthesized single crystals of a number of boracites and showed that they were both ferroelectric and weakly ferromagnetic in some temperature range [43-46]. Full reviews of the existing data and their analysis were reported by Schmid [47] and by Nelmes [48]. A paper by Toledano et al. [49] contains an exhaustive list of references.

The boracites have $F \overline{4} 3 c-T_{d}^{5}$ symmetry in their high temperature phase. In the following, crystal axes referred to the cubic axes will be used. Generally, they have a transition into orthorhombic $P c a 2_{1}-C_{2 v}^{5}$ low-temperature phase at a certain temperature $T_{\mathrm{e}}$, sometimes much higher than room temperature. In a number of cases, ferroelectricity is confirmed in the orthorhombic phase. When the polar $c$ direction of the low-temperature phase is along the [001] axis, the 


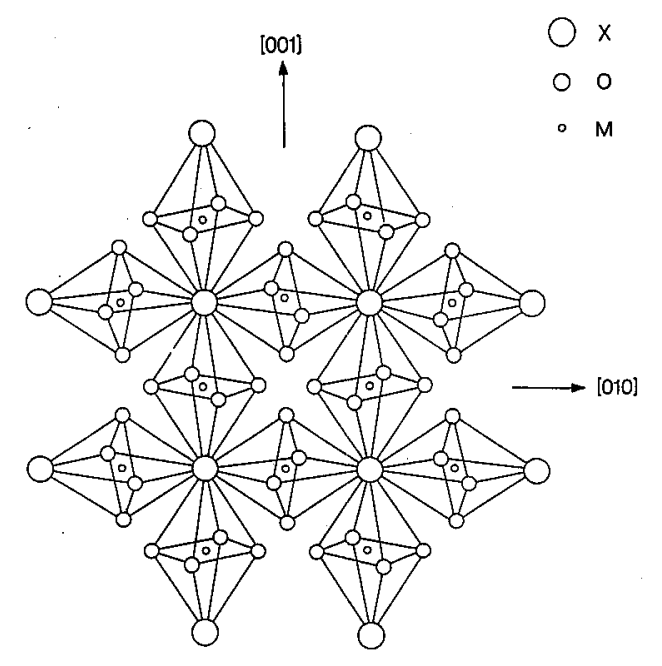

Fig. 11. Crystal structure of boracites.

$a$ - and the $b$-axes are along the [110] and the [110], respectively. $\mathrm{Fe}-\mathrm{Cl}, \mathrm{Fe}-\mathrm{Br}$, $\mathrm{Fe}-\mathrm{I}, \mathrm{Zn}-\mathrm{Cl}$ and $\mathrm{Co}-\mathrm{Cl}$ boracites have another phase with trigonal $R 3 c$ symmetry at lower temperature and a monoclinic one with point group $m 1^{\prime}$ except for $\mathrm{Fe}-\mathrm{Br}$.

The boracites have an ordered magnetic state at low temperatures, when $\mathbf{M}$ is a transition metal. The transition point $T_{\mathrm{m}}$ is generally lower than $T_{\mathrm{e}}$ although the existing data show that they are equal in Ni-I boracite. There is a correlation between $T_{m}$ and the element $\mathrm{X}$, suggesting a superexchange interaction through $\mathrm{X}$ plays an important role in the magnetic ordering.

No definite conclusion is derived on the spin structure. There is a difficulty in neutron diffraction study because of high neutron absorption of boron nuclei. Several experiments seem to support the view that the spin structure is not based on a simple collinear antiferromagnetic type [50-52]. In the cubic boracite structure, metallic ions $M$ are located on three different sublattices in such a way that any inter-sublattice interaction vanishes, if the net magnetic moment of each sublattice is zero. A small distortion from cubic structure is probably playing an essential role in magnetic ordering. The magnetoelectric effect has been utilized to determine magnetic symmetry of the low-temperature phase. The direction of the "parasitic" weak ferromagnetism, accompanied by the magnetic order and determined by Faraday effect and/or magnetic torque studies, too, has given important information on this point.

When we assume that the prototype point symmetry of boracites is $\overline{4} 3 \mathrm{~m}$, sequences of the second-order transition are derived according to the Landau's subgroup rule. They are shown in Fig. 12 with a possible direction of both spontaneous electric and magnetic polarization.

Generally, magnetic boracites are magnetoelectric, i.e., the first-order magnetoelectric effect is allowed. The magnetoelectric effect was observed in many compounds (see Table III). The form of the magnetoelectric tensor gives a clue 


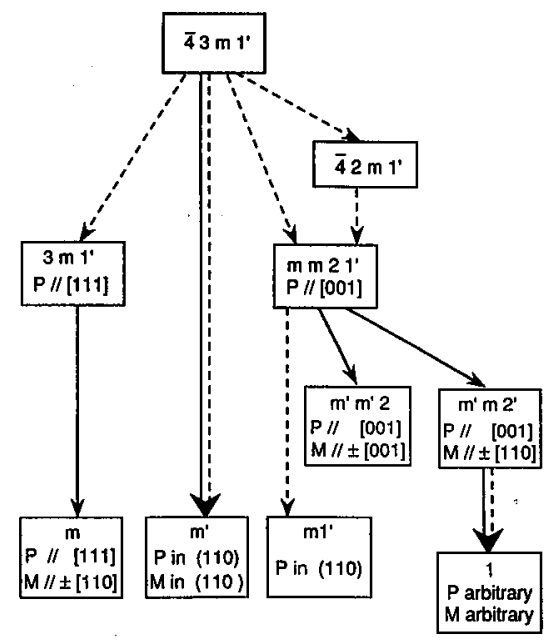

Fig. 12. Subgroups of $\overline{4} 3 m$, which show phase transitions in the boracite family. Solid arrows indicate magnetic transitions and broken arrows indicate structural transitions. Directions of the polarization are also shown.

TABLE III

Magnetic phase transitions in magnetic boracites.

\begin{tabular}{l|l|l}
\hline \hline Material & Magnetic transitions & Refs. \\
\hline $\mathrm{Fe}-\mathrm{Cl}$ & $m-(15 \mathrm{~K})-3 m 1^{\prime}$ & {$[53-55]$} \\
$\mathrm{Fe}-\mathrm{Br}$ & $m(?)-(18 \mathrm{~K})-3 m 1^{\prime}$ & {$[55,56]$} \\
$\mathrm{Fe}-\mathrm{I}$ & $m-(38 \mathrm{~K})-3 m 1^{\prime}$ & {$[57]$} \\
$\mathrm{Co}-\mathrm{Cl}$ & $m-(15 \mathrm{~K})-3 m 1^{\prime}$ & {$[53,55,57]$} \\
$\mathrm{Co}-\mathrm{Br}$ & $m^{\prime} m 2^{\prime}-(17 \mathrm{~K})-m m 21^{\prime}$ & {$[58]$} \\
$\mathrm{Co}-\mathrm{I}$ & $m^{\prime} m 2^{\prime}-(37.5 \mathrm{~K})-m m 21^{\prime}$ & {$[59,60]$} \\
$\mathrm{Ni}-\mathrm{Cl}$ & $m^{\prime} m 2^{\prime}-(9.7 \mathrm{~K})-m m 21^{\prime}$ & {$[25,61-63]$} \\
$\mathrm{Ni}-\mathrm{Br}$ & $1-(21 \mathrm{~K})-m^{\prime} m 2^{\prime}-(29 \mathrm{~K})-m m 21^{\prime}$ & {$[62,64]$} \\
$\mathrm{Ni}-\mathrm{I}$ & $m^{\prime}-(60.5 \mathrm{~K})-m m 21^{\prime}$ & {$[22,43,65,66]$} \\
$\mathrm{Cu}-\mathrm{Cl}$ & $m^{\prime} m^{\prime} 2-(8.5 \mathrm{~K})-m m 21^{\prime}$ & {$[24,67]$} \\
$\mathrm{Cu}-\mathrm{Br}$ & $?-(24 \mathrm{~K})-m m 21^{\prime}$ & {$[56]$}
\end{tabular}

to magnetic symmetry, when combined with the knowledge of the easy direction of weak ferromagnetic moment and polarization, which are often determined with the use of microscopic studies. A part of the results is tabulated in Table III. In most orthorhombic boracites ( $\mathrm{Co}-\mathrm{Br}, \mathrm{Co}-\mathrm{I}, \mathrm{Ni}-\mathrm{Cl}, \mathrm{Ni}-\mathrm{Br}$, etc.), the magnetic class $m^{\prime} m 2^{\prime}$ was concluded from the fact that the spontaneous polarization $\boldsymbol{P}_{0}$ lies along the [001] and the easy axis of magnetization is normal to $\boldsymbol{P}_{0}$. Figure 13 


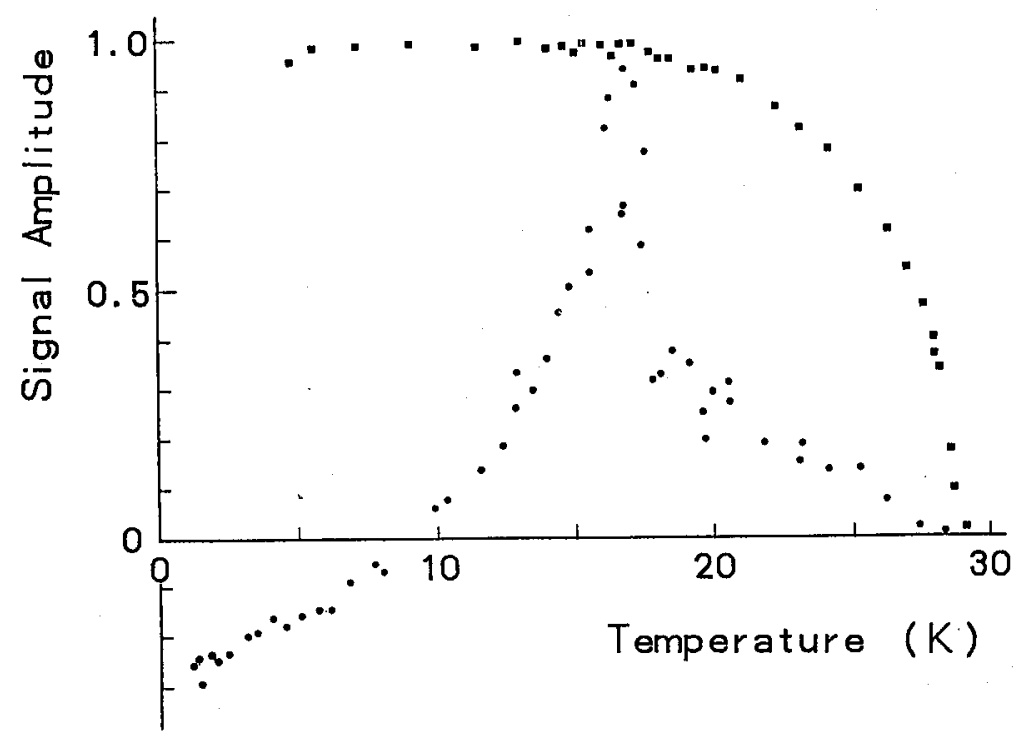

Fig. 13. Temperature dependence of $\alpha_{23}$ (full squares) and $\alpha_{32}(\bullet)$ in Ni-Br boracite $[68,64]$. (Courtesy of H. Schmid).

shows $\alpha_{23}$ and $\alpha_{32}$ in $\mathrm{Ni}-\mathrm{Br}$ boracite which proves two successive transitions. The symmetry of the crystal below $29 \mathrm{~K}$ is $m^{\prime} m 2^{\prime}[68,64]$.

In this family of compounds, Ni-I boracite is unique, because it turns into ferroelectric and weakly ferromagnetic simultaneously at $61.5 \mathrm{~K}$, where the crystal deforms directly from cubic to monoclinic, at variance with other boracites. This transition can be a new type where magnetoelectric interaction plays an important role in the mechanism. Therefore, we will describe this case in detail.

Ferroelectricity of the low-temperature phase of Ni-I boracite was confirmed first by the observation of the dielectric hysteresis loop. Along with this, a small ferromagnetic moment appears. The simultaneous nature of ferroelectric and magnetic transitions was most definitely demonstrated by the measurements of spontaneous polarization, dielectric constant, spontaneous birefringence and ferromagnetic Faraday rotation on the same sample [65].

From the early studies of ferroelectric and weak ferromagnetic domain structure, it was concluded that the spontaneous magnetic moment is along the [110] or [11̄0] direction, according to whether the dielectric polarization $\boldsymbol{P}$ was along the [001] or [001] direction, respectively. The vector $\boldsymbol{P}$ could be switched by the application of the magnetic field within the $c$ plane. This switching was observed as a butterfly-type hysteresis (Fig. 14). Two straight lines crossing the origin correspond to the two kinds of domains. The magnetic symmetry of the low-temperature phase was considered to be $m^{\prime} m 2^{\prime}$. When the direction of polarization was fixed, say, along [001], the sample was in a state of nearly single domain with the magnetic moment along the [110] axis. The bulk magnetoelectric susceptibility $\alpha_{32}$ took either positive or negative value, according to the polarization direction of 


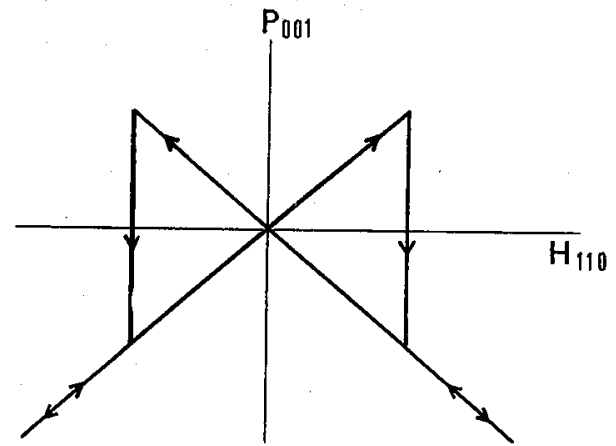

Fig. 14. Butterfly-type hysteresis of the magnetoelectric effect in Ni-I boracite, indicating domain switching by applied magnetic field [47]. (Courtesy of H. Schmid).
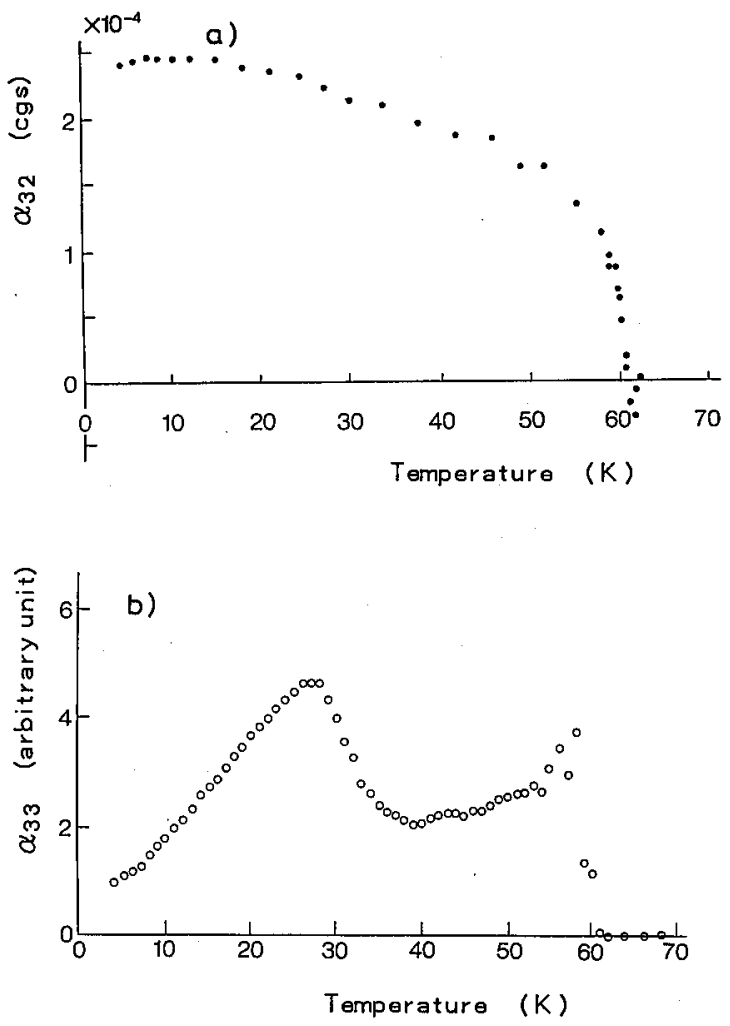

Fig. 15. Temperature dependence of magnetoelectric susceptibilities in Ni-I boracite: (a) $\alpha_{32}$, (b) $\alpha_{33}$. Reproduced with permission from Refs. [66] and [22], respectively. Copyright by Gordon and Breach Science Publishers Ltd. 
the crystal. When the polarization is reversed by an applied electric field, the axis of the easy magnetization is changed by $90^{\circ}$, from [110] to [110] for example. The temperature dependence of $\alpha_{32}$ is shown in Fig. 15a. The change of the sign and a small peak just below $T_{\mathrm{c}}$ was initially considered to be the effect of domains, but not yet found a definite explanation [59].

More detailed studies of the Faraday effect, however, threw doubt on the magnetic class, because strong Faraday rotation was observed for light propagating both along the [001], direction of the poling electric field, and the [110] [65]. This fact means that the spontaneous magnetic moment should have a component along the spontaneous polarization as well as perpendicular to it. Along with this, the index ellipsoid was found to rotate about [110] with temperature. These facts are consistent with the magnetic symmetry $m^{\prime}$, and not with $m^{\prime} m 2^{\prime}[65,66]$. It was verified by the recent detection of $\alpha_{33}$ [22]. The observed temperature dependence of $\alpha_{33}$ is shown in Fig. 15b.

The spin structure of $\mathrm{Ni}-\mathrm{I}$ boracite is still unknown. There are two reports of powder neutron diffraction, whose conclusions contradict each other. Magnetic structure is supposed to be of non-collinear type. One support to this is the fact that none of the susceptibilities along three principal directions tends to zero at $0 \mathrm{~K}$.

The results of the experimentally determined magnetic symmetry of boracites are summarized in Table III. Transitions are classified according to the scheme shown in Fig. 12.

\section{2. $Y I G$}

Yttrium iron garnet, $\mathrm{Y}_{3} \mathrm{Fe}_{5} \mathrm{O}_{12}$, is a ferrimagnetic oxide with magnetic Curie point of $559 \mathrm{~K}$. This is a useful material in magnetics for its optical properties, narrow resonance width and small magnetic anisotropy, which are considered due to the fact that YIG contains only $\mathrm{Fe}^{3+}$ as a transition metal element [69]. The crystal structure has been believed to be cubic $I a \overline{3} d$, though several reports have cast doubts on this point [e.g., 70]. Since $I a \overline{3} d$ has inversion symmetry, no linear magnetoelectric effect but only that of quadratic in the electric field was expected and has been reported [71-73, etc.]. On single crystals prepared with floating zone method, however, first-order magnetoelectric effect has been observed recently, when the crystal is cooled in an external electric field from room temperature to $77 \mathrm{~K}$ [74]. The sign of the signal is reversed by reversing the cooling electric field direction. With increasing temperature, the signal decreases and vanishes at around $125 \mathrm{~K}$, as shown in Fig. 16.

The crystal symmetry of the low-temperature phase of YIG was determined as triclinic, 1 , by the following analysis [75]:

1. The specimen was a disc in the (110) plane, an electric field was applied along the [110] axis and an external magnetic field of several hundred Oe was applied within the (110) plane during cooling of the crystal from room temperature to below $125 \mathrm{~K}$ as well as for the measurement.

2. Two components of the change of the magnetization, due to the electric field, were measured along the [111] and [i112] directions. By the synthesis of the 
two data, it was confirmed that the main part of the effect is the rotation of the magnetization, or the change of the anisotropy energy.

3 . This electrically induced anisotropy causes a torque on the magnetization and the torque was plotted as a function of the direction of the magnetization within the (110) plane. An example is shown in Fig. 17 (solid circles).

4. The torque was analyzed by triangular functions of $2 \phi$ and $4 \phi$ :

Magnetoelectric torque $=A_{2} \sin \left(2 \phi-\alpha_{2}\right)+A_{4} \sin \left(4 \phi-\alpha_{4}\right)$.

Here $\phi$ is the angle between magnetization and the [110]. Four parameters, $A_{2}$, $A_{4}, \alpha_{2}$ and $\alpha_{4}$, were determined with the least-squares fit.

5 . It was disclosed that the phase angle of the $2 \phi$ term, $\alpha_{2}$, was dependent on the direction of the cooling magnetic field, $\xi$, as is shown in Fig. 18, whereas $\alpha_{4}$ and the amplitudes, $A_{2}$ and $A_{4}$, were independent of it.

6. Existence of the electrically induced magnetic anisotropy with finite phase angle $\alpha_{2}$ indicates that the [110] twofold axis or the (001) or (110) mirror plane is absent in the low-temperature phase of YIG. If one of these operations was a symmetry element of the crystal, the magnetization along the [001] direction, for example, could not rotate by the application of the electric field along the [110] axis.

7. Linear magnetoelectric effect with the electric field along the [110] itself confirms that the crystal has electric polarization which has a component along the $[110]$ axis.

8. Points 6 and 7 prove that only " 1 " or the identical operation is the symmetry element of the crystal.

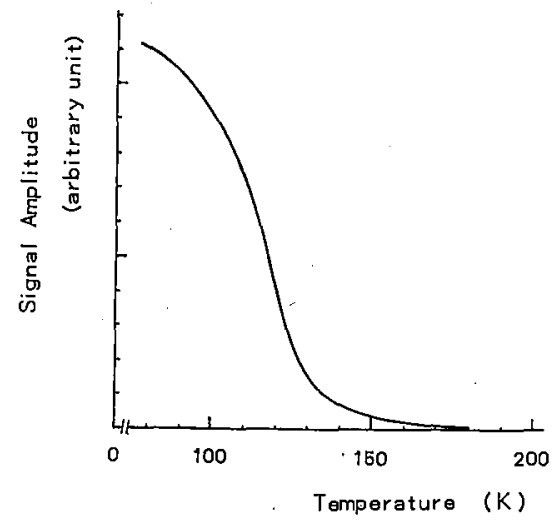

Fig. 16. Temperature dependence of the linear magnetoelectric signal in a single crystal of YIG, cooled to $77 \mathrm{~K}$ in an external electric field. Specimen is a (110) disc. The electric field is applied along the [110] axis and the static magnetic field of 200 Oe along the

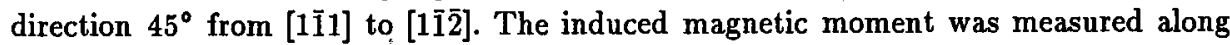
the [111] axis. Reproduced with permission from Ref. [74]. Copyright by the Physical Society of Japan. 


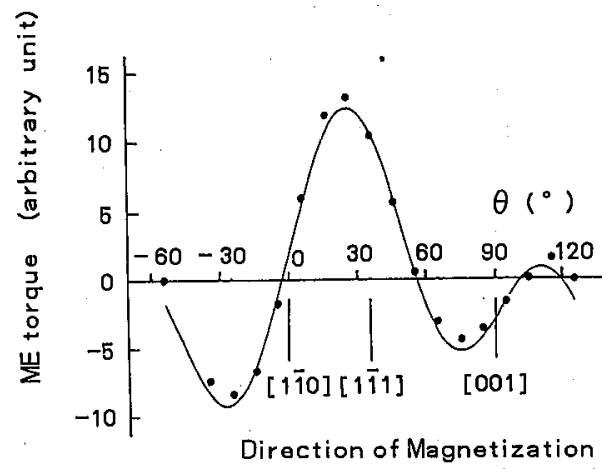

Fig. 17. An example of the electrically induced magnetic torque in the (110) plane, as a function of the magnetization direction (solid circles). The curve is the least-squares fit by Eq. (16). Cooling magnetic field was applied along [111]. Reproduced with permission from Ref. [75]. Copyright by the Physical Society of Japan.

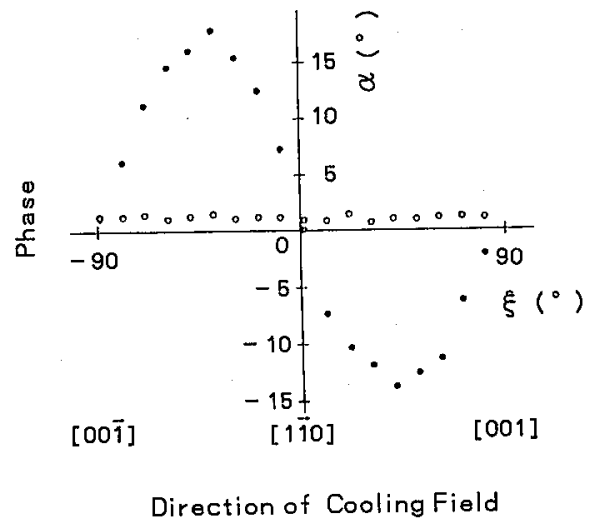

Fig. 18. Dependence of the phase angles, $\alpha_{2}$ and $\alpha_{4}$ in Eq. (16), on the magnetization direction during cooling. Reproduced with permission from Ref. [75]. Copyright by the Physical Society of Japan.

In the above discussion, they considered that a new structural phase, without inversion symmetry, appears below $125 \mathrm{~K}$ in YIG. However, neither diffraction experiments nor Mössbauer spectroscopy have succeeded in finding evidences of structural change so far $[75,76]$. Another possible origin of the linear magnetoelectric effect is the alignment of the electric polarization of the lattice defects, presumably accompanied by the small amount of inevitable reduction of the pure YIG specimens. If this is the case, the above conclusion on the symmetry is not for the crystal lattice but for the defect. The problem whether there is a low-temperature phase in YIG still remains open at present. 


\section{Investigation of the mechanism}

Possible coupling mechanisms between $\boldsymbol{M}$ and $\boldsymbol{P}$, Eq. (9), are the dependence of the exchange interaction and the magnetic anisotropy energy on the ionic displacement and/or the deformation of the electron cloud. Beside these, the dependence of the $g$-factor gives another possibility of magnetoelectric effect, as it was stated before. Quantitative evaluation of the parameters which describe the magnitude of each mechanism is an interesting task of solid state physics and is one of the aims of the investigation of the magnetoelectric effect. Consider, e.g., supposed dependence of the superexchange interaction on the cation-anion-cation bond angle*.

Few attempts of calculation have been made starting from the electronic states of cations ${ }^{\dagger}$. It might be compared with the analysis of paramagnetic resonance by spin Hamiltonian. The parameters in the spin Hamiltonian are determined experimentally and express well the character of that paramagnetic ion. IIowever, quantitative evaluation of the parameter values from the first principle is difficult and not so common, though we trust on the validity of the spin Hamiltonian expression there.

Magnetoelectric effect makes its appearance through the change of the direction and the magnitude of magnetization by applied electric field. The first step of the experimental evaluation of each mechanism is the decomposition of the changing magnetization vector into those parallel and perpendicular to the original magnetization: change of the magnitude and the rotation of the magnetization. The method of the evaluation is dependent on materials, mainly whether they are ferromagnetic or antiferromagnetic.

\subsection{Case of ferromagnets}

Direction of the magnetization of a ferromagnet can be changed rather easily by applying external magnetic field. During the magnetization process due to the $180^{\circ}$ wall motion, the magnetoelectric signal increases proportionally to the magnetization, since both kinds of magnetic domains contribute inversely. During the rotation process, a large signal is expected since the direction of the magnetization is easily changed by the perturbation of the external electric field. After saturation, differential magnetic susceptibility perpendicular to the magnetization decreases almost inversely proportionally to the external magnetic field. If the

* On this point, the effect of transverse displacement of $\mathrm{Ni}^{2+}$ and $\mathrm{F}^{-}$on the superexchange interaction in $\mathrm{KNiF}_{3}$ was discussed $[77,78]$ on the basis of the shift of the infrared absorption line. In this case, anions locate at the symmetric position between cations and no static electric dipole as well as the first-order magnetoelectric effect can be expected.

†Ham [79] analyzed the ESR data of Ludwig and Woodbery [40] on the interstitial transition metal elements in $\mathrm{Si}$. Royce and Bloembergen [42] measured the electric shift of the spin Hamiltonian parameters of $\mathrm{Cr}^{3+}$ in ruby and discussed its electronic origin. In both cases, it was concluded that the main source of the effect is the modification of the molecular orbitals of $3 d$ electrons of the transition metal by the electric field. In ruby, relative shift of cations and anions was considered important for the modification. A similar consideration seems difficult for the exchange coupling constants, since the theory of superexchange interaction has not been developed so well. 


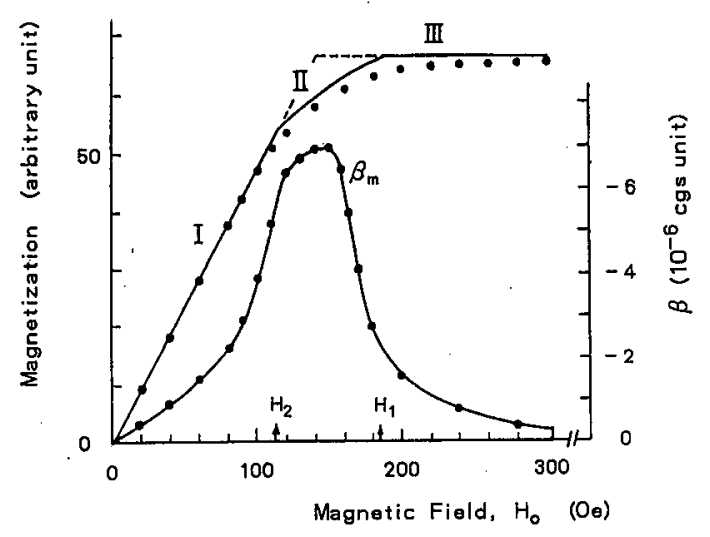

Fig. 19. Dependence of the magnetization and the second-order magnetoelectric effect on the external magnetic field, during the technical magnetization process of YIG at room temperature. Region I is of the domain wall motion, II is of rotation and III is of saturation. Reproduced with permission from Ref. [73]. Copyright by Gordon and Breach Science. Publishers Ltd.

magnetoelectric effect is due to the rotation of the magnetization, the magnetoelectric susceptibility should be inversely proportional to the external magnetic field. Qualitatively, these features were confirmed during the technical magnetization process of YIG, for the magnetoelectric effect proportional to $E^{2}$ [73] (see Fig. 19). It should be pointed out that quantitative explanation of the magnetoelectric effect in YIG was given later by the same group [80].

For the quantitative analysis, the data obtained during the technical magnetization process is not so convenient. After saturation, the system is homogeneous and magnetic susceptibility $\chi$, as well as the magnetization direction can be easily evaluated. In order to know the induced moment fully, the detection should be made along three different directions. An example for YIG was presented in the last section. Figures 20 and 21 show another example of $\mathrm{Fe}_{3} \mathrm{O}_{4}^{\prime}$ [81]. The signal amplitude plotted against the inverse of the curvature of energy (Zeeman + anisotropy) or perpendicular differential susceptibility should be a straight line. The slope of the line gives induced rotation of the magnetization and the value extrapolated to the origin of the ordinate gives change of the magnitude of the moment. In the case shown in Fig. 21, there is only the rotation of the magnetization due to the applied electric field. By Fourier transformation of the induced rotation as a function of the magnetization direction, magnetoelectric anisotropy energy can be analyzed as ordinary magnetic anisotropy energy. In the case of the first-order effect in the (110) plane of YIG, $2 \phi$ and $4 \phi$ terms were sufficient as mentioned in the last section.

Direct measurements of the magnetic anisotropy have been reported, too, by torque or resonance. Figure 22 shows a torque curve in the (110) plane of YIG at $77 \mathrm{~K}$, with and without electric field [82]. The difference of two torque curves 

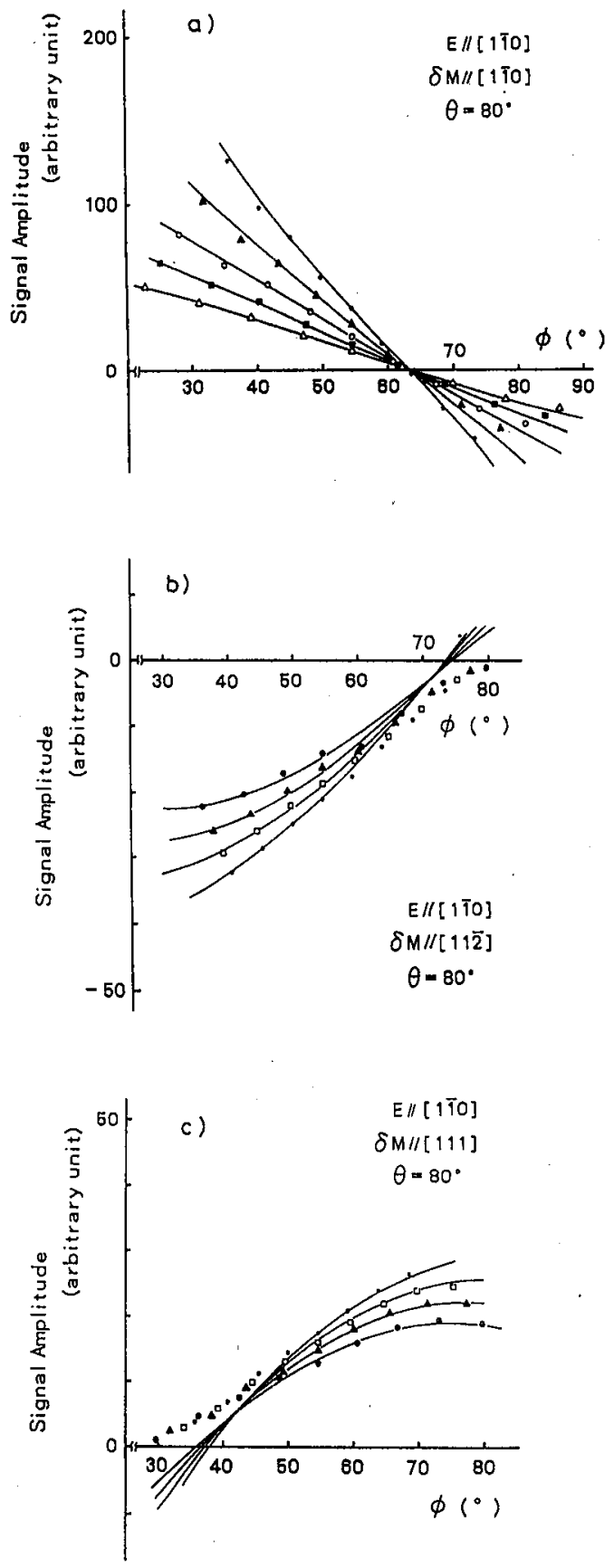

Fig. 20. Dependence of the magnetoelectric signal amplitude along three different axes on the magnetization direction, in the low-temperature phase of $\mathrm{Fe}_{3} \mathrm{O}_{4}$ at $77 \mathrm{~K} . \theta$ and $\phi$ are polar and azimuthal angles of magnetization from [110] and [111], respectively. Reproduced with permission from Ref. [81]. Copyright by the Physical Society of Japan. 


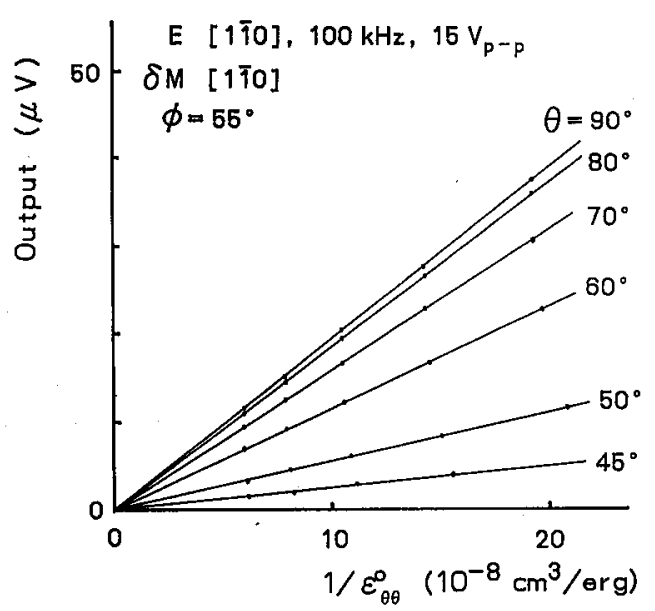

Fig. 21. Plots of signals in Fig. 20 against the second derivatives of Zeeman + anisotropy energy on behalf of the polar angle of magnetization, for $\phi=55^{\circ}$. The ordinate indicates "susceptibility" of the magnetization rotation . Reproduced with permission from Ref. [81]. Copyright by the Physical Society of Japan.

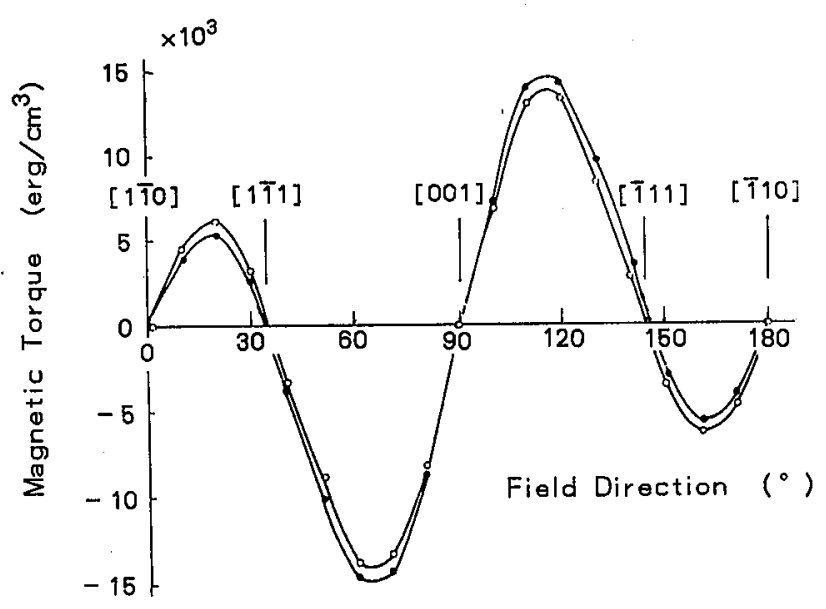

Fig. 22. Magnetic torque curves in the (110) plane of a YIG single crystal at $77 \mathrm{~K}$, with $(\bullet)$ and without (0) electric field of $11 \mathrm{kV} / \mathrm{cm}$ along [110]. Magnetic field was $20 \mathrm{kOe}$. Reproduced with permission from Ref. [82]. Copyright by the Academy of Sciences Press.

could be expressed by an anisotropy energy

$$
\Delta L=-1.2 \times 10^{3} \sin 2 \theta \quad\left[\mathrm{erg} / \mathrm{cm}^{3}\right],
$$

when an electric field of $11 \mathrm{kV} / \mathrm{cm}$ is applied along the [110] axis. After the removal of the external electric field, the torque did not return to the original form. This fact suggests that the effect is linear in $E$, though dependence on the electric 
field strength was not reported. It is to be noted that the magnetic torque is caused not only by the energy dependent on the magnetization direction (ordinary anisotropy energy), but also by the anisotropy of the $g$-factor or the magnitude of the magnetization. To separate these mechanisms, the electrically induced torque should be measured as a function of the measuring magnetic field.

The shift of the ferromagnetic resonance due to the static electric field was measured for ordered $\mathrm{LiFe}_{5} \mathrm{O}_{8}$ [83] and its temperature dependence is shown in Fig. 23. Experimental data (points) are compared with the value calculated from

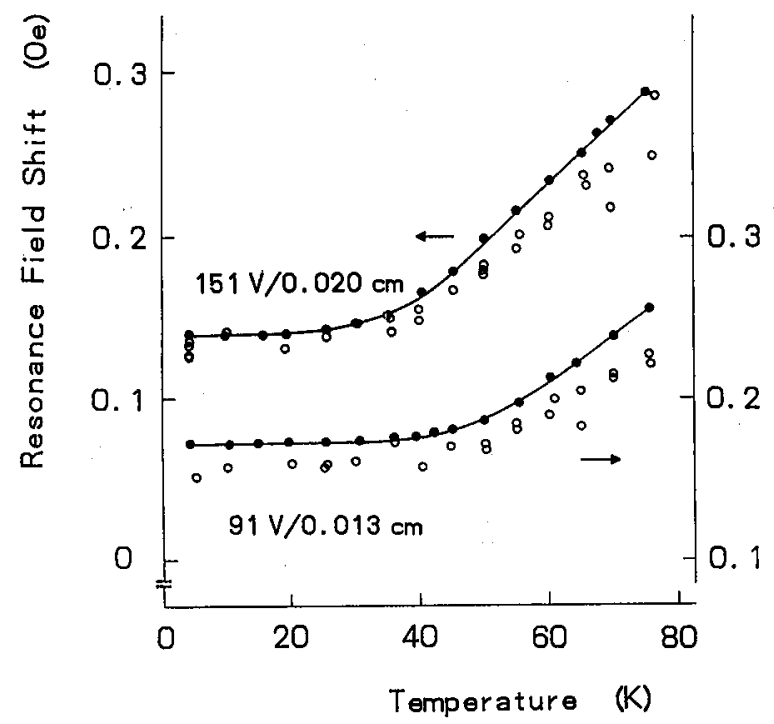

Fig. 23. Temperature dependence of the electric field shift of the ferromagnetic resonance field at $10 \mathrm{GHz}$ for ordered $\mathrm{LiFe}_{5} \mathrm{O}_{8}$ (points), compared with that calculated from the data of static measurements. Reproduced with permission from Ref. [83]. Copyright by the American Physical Society.

that of static measurements by the same authors (solid lines). The latter experiment was performed by detecting electric polarization induced by the change of the magnetization direction and the data was analyzed assuming uniaxial magnetic anisotropy energy linear to the electric field. Note that the transition temperature of the order-disorder transformation in $\mathrm{LiFe}_{5} \mathrm{O}_{8}$ is as high as $\sim 1030 \mathrm{~K}$. The specimen was prepared with flux method and no magnetoelectric cooling was applied before the experiment [84]. The signal of the magnetoelectric effect was reported to be dependent on the position (whether solidified at the beginning of the crystallization or not) in a single crystal.

As reported so far, the main mechanism of magnetoelectric effect in the ferromagnets is the rotation of the magnetization due to the electric field, caused by the change of the anisotropy energy. 


\subsection{Case of $\mathrm{Cr}_{2} \mathrm{O}_{3}$}

In the case of collinear antiferromagnet, the separation of the effect into rotation and non-rotation parts is rather easy, since the magnetization lies along the easy axis and does not change its direction so much*. For example, $\alpha_{11}$ in Eq. (2) for $\mathrm{Cr}_{2} \mathrm{O}_{3}$ gives perpendicular part and $\alpha_{33}$ is for parallel. This material will be described in this subsection as a typical example.

The temperature dependence of $\alpha_{33}$ shown in Fig. 2 has a characteristic form: at low temperatures the magnitude of $\alpha_{33}$ is small, but finite and almost constant. The absolute value decreases with increasing temperature and changes its sign at around $90 \mathrm{~K}$ and vanishes at $T_{\mathrm{N}}$ of about $300 \mathrm{~K}$ after a rather sharp maximum at around $260 \mathrm{~K}$. This profile suggests that the effect is proportional to $M \chi$, i.e. mainly due to $\delta S$, a change of the thermal average of spins in a sublattice, but $\delta g$, a change of the $g$-factor, exists, too, in the opposite direction. In an ordinary collinear antiferromagnet, magnetic susceptibility along the easy axis decreases with decreasing temperature and vanishes at $0 \mathrm{~K}^{\dagger}$. The origin of $\delta S$ was attributed to the change of the intra-sublattice exchange interaction, since the change of the uniaxial anisotropy energy, estimated from the effect in ruby, was too small to account for the observed magnitude [41].

This point was studied further by Hornreich and Shtrikman [11]. They adopted molecular field theory of the antiferromagnet with uniaxial anisotropy and treated the anisotropy energy as a perturbation to the molecular field. They deduced the following expression of the electrically induced change of the thermal average of spins:

$$
2 N \mu_{\mathrm{B}} g_{0} \delta S=\frac{\chi_{33} S_{0}}{g_{0} \mu_{\mathrm{B}}}\left\{-\delta \Gamma+\delta D \frac{\left\langle S_{z}^{3}\right\rangle-\left\langle S_{z}^{2}\right\rangle\left\langle S_{z}\right\rangle}{\left\langle S_{z}\right\rangle\left[\left\langle S_{z}^{2}\right\rangle-\left\langle S_{z}\right\rangle^{2}\right]}\right\}
$$

Here, $\langle *\rangle$ is the thermal average of quantity $*$ and $\Gamma$ stands for the molecular field constant of intra-sublattice exchange interactions. The factor after $\delta D$ makes temperature dependence of the effect different from those caused by the change of the intra-sublattice exchange coupling and of the anisotropy energy (see Fig. 24). The former gives better coincidence with experiment. It was indicated that $\delta \Gamma$ or $\delta D$ should be about $4 \times 10^{-7} \mathrm{~cm}^{-1} \cdot(\mathrm{V} / \mathrm{cm})^{-1}$, in order to account for the peak value of $\alpha_{33}$. This magnitude of the latter should be compared with $\delta D=$ $6 \times 10^{-9} \mathrm{~cm}^{-1} \cdot(\mathrm{V} / \mathrm{cm})^{-1}$, observed for $\mathrm{Cr}^{3+}$ ions in ruby [42].

Such an analysis is based on an assumption that coupling parameters are not dependent on temperature. At the same time, approximation in statistical mechanics will affect the results. If we want to avoid these difficulties, the analysis should be carried out for the data at one temperature, not for their temperature dependence. Apparently, experimental data other than $\alpha_{33}$ is necessary. It was shown that antiferromagnetic resonance meets these needs.

*This last point is a demerit for the investigation, compared with ferromagnet. External magnetic field higher than the critical field for the spin-flipping can change the direction of sublattice magnetizations [85], though the range of the direction is not so wide [86] and experiment has been scarce.

$\dagger$ In the case of $\mathrm{Cr}_{2} \mathrm{O}_{3}, \chi_{\|}$was reported to be finite at $\mathrm{OK}$ [87]. If this is due to the Van Vleck term [11], the following argument can be adopted. See also Ref. [88]. 


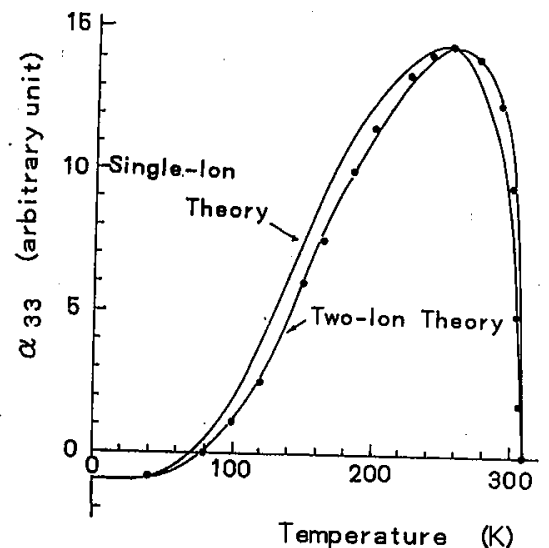

Fig. 24. Temperature dependence of $\alpha_{33}$ of $\mathrm{Cr}_{2} \mathrm{O}_{3}$, compared with the calculation by the molecular field approximation. Points are the experimental data of Astrov [5]. Reproduced with permission from Ref. [11]. Copyright by the American Physical Society.

Resonance frequency of an uniaxial antiferromagnet is determined by the inter-sublattice exchange energy, $A$, Zeeman energy and the anisotropy energy as

$$
\frac{E}{N}=A\left(\boldsymbol{S}_{\downarrow} \cdot S_{\uparrow}\right)-\mu_{\mathrm{B}} \boldsymbol{H} \cdot\left[g_{\uparrow} \boldsymbol{S}_{\uparrow}+g_{\downarrow} S_{\downarrow}\right]-\left[D_{\uparrow} S_{\uparrow z}^{2}+D_{\downarrow} S_{\downarrow z}^{2}\right] .
$$

In this equation, $g, D$ and $S$, thermal average of the sublattice magnetization, change by the externally applied electric field. The shift of the resonance frequency proportional to the electric field can be derived easily as

$$
\begin{aligned}
& \hbar \omega=\hbar \omega_{0}+\hbar \delta \omega \\
& =\sqrt{4\left(A+D_{0}\right) D_{0} S_{0}^{2}} \pm g \mu_{\mathrm{B}} H \pm\left[\left(2 D_{0}-A\right) \cdot \delta S+2 S_{0} \delta D\right] \\
& +\frac{\left(A+2 D_{0}\right) \mu_{\mathrm{B}} H}{\sqrt{4\left(A+D_{0}\right) D_{0}}} \delta g .
\end{aligned}
$$

Equation (20) shows that the shift due to $\delta S$ and $\delta D$ is independent of $H$, being constant for each branch of the resonance. On the other hand, $\delta g$ gives a shift proportional to $H$. Thus, measurements of $\delta \omega$ at different values of the magnetic field $H$ or the frequency $\omega$ enable us to distinguish the effect of $\delta g$. Then, $\delta S$ can be determined from the observed $\alpha_{33}$ and $\delta D$ from Eq. (20).

The experiment has been carried out only at $24.2 \mathrm{GHz}$ at $4.2 \mathrm{~K}$ [89]. An ac electric field was applied along the $c$ axis at $100 \mathrm{kHz}$ and the modulated signal of antiferromagnetic resonance was observed and compared with the signal observed by the magnetic field modulation. Traces of the signal are shown in Fig. 25. The result was

$$
\hbar \delta \omega / E=-3.4 \times 10^{-6} \mathrm{~cm}^{-1} \cdot(\mathrm{kV} / \mathrm{cm})^{-1},
$$

after the magnetoelectric cooling along the $c$ axis with parallel electric and magnetic fields. If we assume that $\delta S=0$ at this temperature, $\alpha_{33}$ of $-1.2 \times 10^{-6} \mathrm{cgs} / \mathrm{g}$ (see Fig. 2), measured for the same crystal, gives

$$
\delta g=-3.5 \times 10^{-8} \mathrm{~cm}^{-1} \cdot(\mathrm{kV} / \mathrm{cm})^{-1}
$$




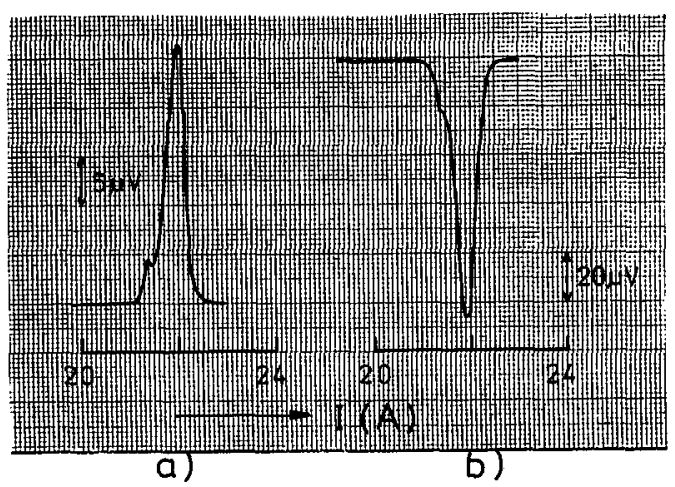

Fig. 25. Recorder trace of the antiferromagnetic resonance of $\mathrm{Cr}_{2} \mathrm{O}_{3}$ at $24.2 \mathrm{GHz}$, 4.2 K. Static magnetic field as well as modulation fields were applied along the $c$ axis. Experiment of electric field modulation was performed after the parallel magnetoelectric cooling along the $c$ axis. (a) Signal by electric field modulation, $360 \mathrm{~V}_{\mathrm{p}-\mathrm{p}} / 0.4 \mathrm{~mm}$. (b) Signal by magnetic field modulation, $0.9 \mathrm{Oe}_{\mathrm{p}-\mathrm{p}}$. Reproduced with permission from Ref. [89]. Copyright by the Physical Society of Japan.

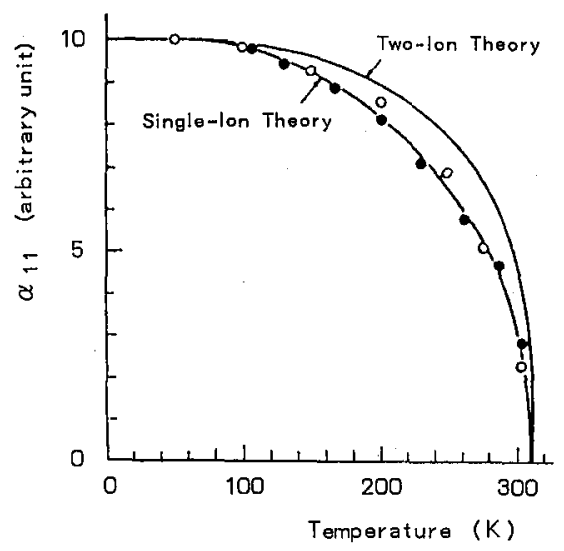

Fig. 26. Analysis of the temperature dependence of $\alpha_{11}$ in $\mathrm{Cr}_{2} \mathrm{O}_{3}$. Open circles are after Astrov [5] and solid circles are after Folen et al. [6] . Reproduced with permission from Ref. [11]. Copyright by the American Physical Society.

and, according to Eq. (21),

$$
\delta D=-1.1 \times 10^{-6} \mathrm{~cm}^{-1} \cdot(\mathrm{kV} / \mathrm{cm})^{-1} .
$$

This magnitude is more than two orders too small to account for the peak value of $\alpha_{33}$. Moreover, the sign should be reversed.

In contrast to $\alpha_{33}$, the temperature dependence of $\alpha_{11}$ is similar to that of $M$, sublattice magnetization. This is natural since $\chi_{\perp}$, magnetic susceptibility of an antiferromagnet perpendicular to the spin axis, is nearly constant below 
the Néel point. Hornreich and Shtrikman argued on the quantitative analysis of the temperature dependence by the molecular field theory that the tilting of the anisotropy axis causes magnetoelectric effect in this case [11] (see Fig. 26). No experiment of antiferromagnetic resonance has been carried out for the electric field within the $c$ plane.

\section{Optical observation}

Propagation of electromagnetic waves in a magnetoelectric medium was discussed by several authors. Fuchs [90] treated the orthorhombic case such as 222 (see Table I). It was shown that the phase velocity of light is determined by a quartic equation, coefficients of which are functions of electric, magnetic and magnetoelectric susceptibilities. Waves propagating along a general direction, not parallel to the principal axes, show non-reciprocity, i.e., the phase velocity is different for two waves with opposite direction. Birefringence appears in light waves along one principal axis. It was argued that the observation of these effects at near-infrared region would be effective to distinguish the origin, whether atomic displacement or distortion of the electron cloud is responsible. Atomic displacement cannot respond to the electric field of light if the frequency is higher than the lattice vibration.

This argument is logically correct but rather misleading. Firstly, the main term of the magnetoelectric effect is due to the change of the thermal average of magnetization, in its magnitude and direction, due to the applied electric field. To observe the effect, the frequency of the electric field should be lower than the inverse of the relaxation time of spins. This threshold is much lower than the lattice vibration. Only the small effect caused by the change of the $g$-factor might be valid here. Secondly, magnetic materials have several mechanisms of birefringence or non-reciprocity. It is hard to evaluate the small effect due to the magnetoelectric effect, though that might be possible, in principle, by observing the difference for the magnetoelectric cooling with opposite field direction. There has been no observation of the magnetoelectric effect in the optical region.

The highest frequency of the magnetoelectric effect observation is the order of $\mathrm{MHz}$, so far. The noise due to the direct coupling between the excitation and the detection circuits increases with increasing frequency faster than the signal, resulting in the decrease of the $\mathrm{S} / \mathrm{N}$ of the measurement. As for the relaxation, a Richter type decrease of the signal with the relaxation time of $2 \mu$ s was reported for $\mathrm{Fe}_{3} \mathrm{O}_{4}$ at $77 \mathrm{~K}$ [17] (see Fig. 27). Of course, the relaxation time will depend on materials and temperature. However, there has been no other report on the relaxation of the magnetoelectric effect. Because of the appearance of the magnetization, external electric field is expected to induce non-reciprocal optical effect such as Faraday effect, in the magnetoelectric antiferromagnet. This was observed by Pisarev's group for $\mathrm{Cr}_{2} \mathrm{O}_{3}$ [16]. Figure 28 shows the temperature dependence of the specific non-reciprocal rotation of the plane of polarization (solid line), proportional to the external electric field. Propagation of light as well as the electric field were parallel to the $c$ axis. Apparently, this curve is different from that of $\alpha_{33}$ in Fig. 2. Krichevtsov et al. demonstrated that the quantity could be divided into two parts, one is proportional to the sublattice magnetization (the broken line in Fig. 28) and 


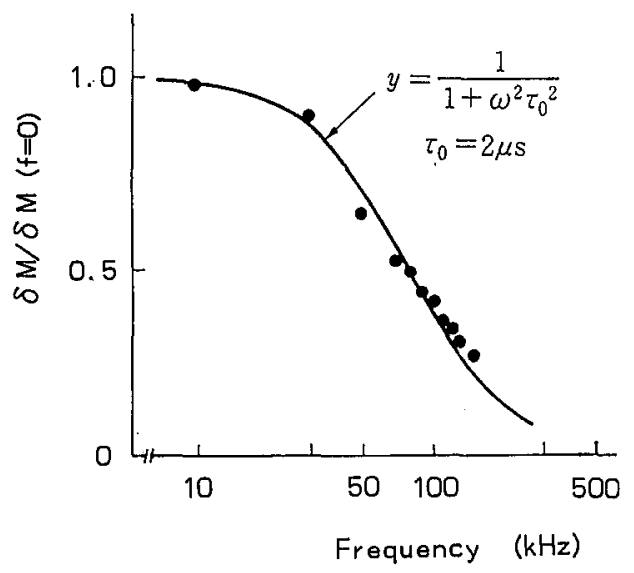

Fig. 27. Frequency dependence of the magnetoelectric effect in $\mathrm{Fe}_{3} \mathrm{O}_{4}$ at $77 \mathrm{~K}$. $E\|[1 \overline{1} 0], \delta M\|[1 \overline{1} 0], H \|[001]$. The solid curve is for the case of single relaxation time of $2 \mu$ s. Reproduced with permission from Ref. [17]. Copyright by the Physical Society of Japan.

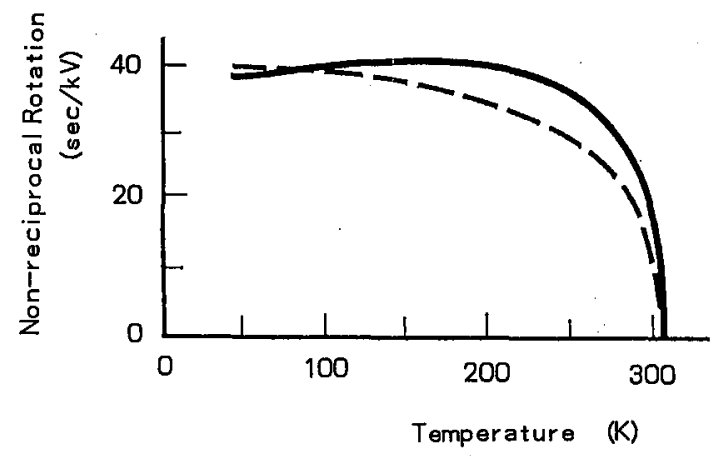

Fig. 28. Temperature dependence of the specific non-reciprocal rotation of the plane of polarization of light in $\mathrm{Cr}_{2} \mathrm{O}_{3}$ (solid line), proportional to the external electric field. Direction of both the light propagation and the electric field are parallel to the $c$ axis. Solid curve: experimental observation. Broken curve: sublattice magnetization. Reproduced with permission from Ref. [16]. Copyright by the Academy of Sciences of USSR.

another to $\alpha_{33}$, as shown in Fig. 29. They pointed out that such a decomposition is reasonable since $\left(M_{\uparrow z}-M_{\downarrow z}\right) \cdot E_{z}$ and $\left(M_{\uparrow z}+M_{\downarrow z}\right)$ are transformed just in the same way by the symmetry operations of the $\mathrm{Cr}_{2} \mathrm{O}_{3}$ crystal. Note that $\left(M_{\uparrow z}-M_{\downarrow z}\right)$ is just the sublattice magnetization.

Existence of non-reciprocal rotation of the polarization plane proportional to $\left(M_{\uparrow z}-M_{\downarrow z}\right) \cdot E_{z}$ suggests that the electric field along the $c$ axis affects inversely to (some) optical absorption of $\mathrm{Cr}^{3+}$ ions in [+] and [-] sites. This had been reported in 1961 for the fluorescence of $R$ lines of ruby [91]. The splitting proportional to the 


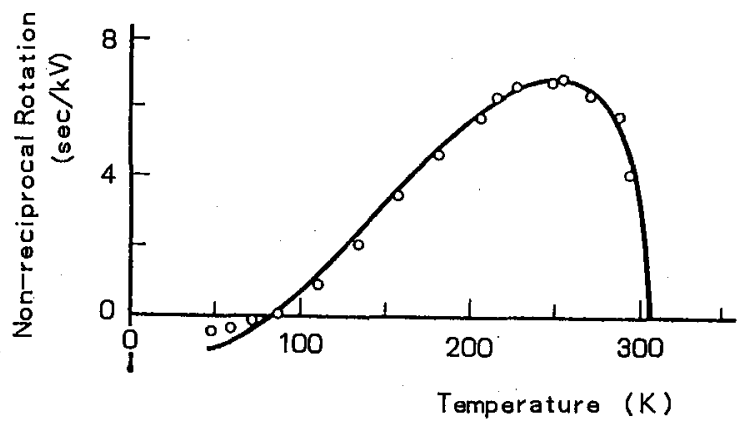

Fig. 29. Non-reciprocal rotation of polarization proportional to magnetoelectric susceptibility $\alpha_{33}$ was separated from that proportional to sublattice moment (see Fig. 28). Solid circles show temperature dependence of $\alpha_{33}$ after Astrov [5] . Reproduced with permission from Ref. [16]. Copyright by the Academy of Sciences of USSR.

electric field, magnitude of which was about $1 \mathrm{~cm}^{-1}$ at $E_{z}=1.7 \times 10^{5} \mathrm{~V} / \mathrm{cm}$ was observed. This is not ordinary Stark splitting, since electric field cannot remove the degeneracy of Kramers doublets; the initial and the final states of the $R$ emission. Optical measurements will supply informations on the magnetoelectric properties of excited state.

\section{Acknowledgements}

The authors would like to express their sincere thanks to Prof. H. Schmid of the University of Geneva for critical reading and comments on the manuscript, especially for the part on boracites, and showing them the results of their most recent experiments.

\section{References}

[1] J.H. Van Vleck, The Theory of Electric and Magnetic Susceptibilities, Oxford University Press, Oxford 1932.

[2] L.D. Landau, E.M. Lifshitz, Elektrodinamika Sploshnuikh Sred, Gosudarstvennoe Izdatel'stvo Fiziko-Matematicheskoi Literatury, Moscow 1957.

[3] I.E. Dzyaloshinski, Zh. Eksp. Teor. Fiz. 37, 881 (1959).

[4] D.N. Astrov, Zh. Eksp. Teor. Fiz. 38, 984 (1960).

[5] D.N. Astrov, Zh. Eksp. Teor. Fiz. 40, 1035 (1961).

[6] V.J. Folen, G.T. Rado, E.W. Stalder, Phys. Rev. Lett. 6, 607 (1961).

[7] I.E. Dzyaloshinski, J. Phys. Chem. Solids 4, 241 (1958).

[8] Magnetoelectric Interaction Phenomena in Crystals, Eds. H. Schmid, J. Freeman, Gordon \& Breach, London 1975.

[9] T.H. O'Dell, The Electrodynamics of Magneto-electric Media, North-Holland, London 1970.

[10] T. Moriya, in: Magnetism, Eds. G.T. Rado, H. Suhl, Vol. I, Academic Press, New York 1963, Ch. 3, pp. 85-125. 
[11] R.M. Hornreich, S. Shtrikman, Phys. Rev. 161, 506 (1967).

[12] E. Kita, A. Tasaki, K. Siratori, Jpn. J. Appl. Phys. 18, 1361 (1979).

[13] C.G. Shull, W.A. Strauser, B.C. Wollan, Phys. Rev. 83, 333 (1951).

[14] H. Kondoh, T. Takeda, J. Phys. Soc. Jpn. 19, 2041 (1964).

[15] T. Yamada, S. Saito, Y. Shimomura, J. Phys. Soc. Jpn. 21, 672 (1966).

[16] B.B. Krichevtsov, V.V. Pavlov, R.V. Pisarev, Zh. Eksp. Teor. Fiz. 94, 284 (1988).

[17] K. Siratori, E. Kita, G. Kaji, A. Tasaki, S. Kimura, I. Shindo, K. Kohn, J. Phys. Soc. Jpn. 47, 1779 (1979).

[18] C.A. Brown, T.H. O'Dell, IEEE Trans. Magn. (USA) MAG-5, 964 (1969).

[19] Yu.A. Izumov, Zh. Eksp. Teor. Fiz. 42, 1673 (1962).

[20] A.W. Overhauser, Bull. Am. Phys. Soc. 7, 241 (1962).

[21] K. Siratori, J. Akimitsu, E. Kita, M. Nishi, J. Phys. Soc. Jpn. 48, 1111 (1980).

[22] Y. Iguchi, K. Kohn, Ferroelectrics, in press.

[23] A.N.N. Christensen, S. Quezel, Solid State Commun. 10, 765 (1972).

[24] M. Haida, K. Kohn, J. Kobayashi, J. Phys. Soc. Jpn. 39, 1625 (1975).

[25] J.P. Rivera, H. Schmid, J.P. Moret, H. Bill, Int. J. Magn. 6, 211 (1974).

[26] G.T. Rado, Phys. Rev. Lett. 23, 644 (1969).

[27] R.R. Birss, Symmetry and Magnetism, North-Holland, Amsterdam 1964.

[28] G.T. Rado, V.J. Folen, Phys. Rev. Lett. 7, 310 (1961).

[29] L.D. Landau, E.M. Lifshitz, Statisticheskaya Fizika, Gosudarstvennoe Izdatel'stvo Fiziko-Matematicheskoì Literatury, Moscow 1951.

[30] G. Gorodetsky, R.M. Hornreich, S. Shtrikman, Phys. Rev. Lett. 31, 938 (1973).

[31] R.M. Hornreich, H.J. Scheel, B. Sharon, Phys. Rev. B 16, 1112 (1977).

[32] M. Mercier, G. Velleaud, J. Phys. Lett. (Paris) 36, L-251 (1975).

[33] L.M. Holmes, L.G. Van Uitert, G.W. Hull, Solid State Commun. 9, 1373 (1971).

[34] G. Gorodetsky, R.M. Hornreich, B.M. Wanklyn, Phys. Rev. B 8, 2263 (1973).

[35] G.T. Rado, Solid State Commun. 8, 1349 (1970).

[36] A.H. Cooke, S.J. Swithenby, M.R. Wells, Int. J. Magn. 4, 309 (1973).

[37] E. Fisher, G. Gorodetsky, S. Shtrikman, J. Phys. (Paris) 32, C1-650 (1971).

[38] A.B. Roitsin, Usp. Fiz. Nauk 105, 677 (1972).

[39] W.B. Mims, The Linear Electric Field Effect in Paramagnetic Resonance, Clarendon Press, Oxford 1976.

[40] G.W. Ludwig, H.H. Woodbery, Phys. Rev. Lett. 7, 240 (1961).

[41] M. Date, J. Kanamori, M. Tachiki, J. Phys. Soc. Jpn. 16, 2589 (1961).

[42] E.B. Royce, N. Bloembergen, Phys. Rev. 131, 1912 (1963).

[43] E. Ascher, H. Rieder, D. Tar, Solid State Commun. 2, 45 (1964).

[44] E. Ascher, H. Rieder, H. Schmid, H. Stossel, J. Appl. Phys. 37, 1404 (1966).

[45] H. Schmid, J. Phys. Chem. Solids 26, 973 (1965).

[46] H. Schmid, H. Rieder, E. Ascher, Solid State Commun. 3, 327 (1965).

[47] H. Schmid, Growth of Crystals, Vol. 7, Consultants Bureau, New York 1969, p. 25. 
[48] R.J. Nelmes, J. Phys. C 7, 3840 (1974).

[49] P. Toledano, H. Schmid, M. Clin, P. Rivera, Phys. Rev. B 32, 6006 (1985).

[50] W. von Wartburg, Phys. Status Solidi A 21, 557 (1974).

[51] W. Schäfer, G. Will, Phys. Status Solidi A 28, 211 (1975).

[52] V.P. Plakhtii, A.V. Kovalev, M.N. Bedrizova, I.V. Golosovskii, G.T. Andreeva, Fiz. Tverd. Tela 18, 2030 (1976).

[53] H. Schmid, Int. J. Magn. 4, 337 (1973).

[54] M. Haida, K. Kohn, 1973, unpublished.

[55] L.N. Baturov, B.I. Al'shin, D.N. Astrov, Fiz. Tverd. Tela 19, 916 (1977).

[56] G. Quezel, H. Schmid, Solid State Commun. 6, 447 (1968).

[57] M. Murata, M. Haida, K. Kohn, 1974, unpublished.

[58] M. Clin, J.P. Rivera, H. Schmid, Ferroelectrics 79, 173 (1988).

[59] M. Clin, J.P. Rivera, H. Schmid, Ferroelectrics 108, 213 (1990).

[60] M. Clin, H. Schmid, P. Shobinger, P. Fischer, Phase Transit. 33, 149 (1991).

[61] M. Haida, K. Kohn, H. Schmid, J. Phys. Soc. Jpn. 37, 1463 (1974).

[62] I.H. Brunskill, H. Schmid, Ferroelectrics 36, 395 (1981).

[63] J.P. Rivera, H. Schmid, J. Appl. Phys. 70, 6410 (1991).

[64] J.P. Rivera, F.J. Schäfer, W. Kleemann, H. Schmid, Jpn. J. Appl. Phys. Suppl. 24-2, 1060 (1985).

[65] J.P. Rivera, H. Schmid, Ferroelectrics 36, 447 (1981).

[66] M. Clin, J.P. Rivera, H. Schmid, Ferroelectrics 108, 207 (1990).

[67] J.P. Rivera, H. Schmid, J. Phys. (France) C-8, 849 (1988).

[68] J.P. Rivera, H. Schmid, Ferroelectrics 55, 295 (1984).

[69] G. Winkler, Magnetic Garnets, Vieweg, Braunschweig 1981.

[70] J. Dong, K. Lu, Phys. Rev. B 43, 8808 (1991).

[71] T.H. O'Dell, Philos. Mag. 16, 487 (1967).

[72] M.J. Cardwell, Philos. Mag. 20, 1087 (1969).

[73] M. Mercier, Int. J. Magn. 6, 77 (1974).

[74] H. Ogawa, E. Kita, Y. Mochida, K. Kohn, S. Kimura, A. Tasaki, K. Siratori, J. Phys. Soc. Jpn. 56, 452 (1987).

[75] S. Takano, E. Kita, K. Siratori, K. Kohn, S. Kimura, A. Tasaki, J. Phys. Soc. Jpn. 58, 1145 (1989).

[76] S. Hirakata, M. Tanaka, K. Kohn, E. Kita, K. Siratori, S. Kimura, A. Tasaki, J. Phys. Soc. Jpn. 60, 294 (1991).

[77] K. Sintani, Y. Tomono, A. Tsuchida, K. Siratori, J. Phys. Soc. Jpn. 25, 99 (1968).

[78] Y. Tomono, T. Takaoka, M. Yajima, Y. Tanokura, N. Jinda, J. Phys. Soc. Jpn. 59, 579 (1990).

[79] F.S. Ham, Phys. Rev. Lett. 7, 242 (1961).

[80] G. Velleaud, B. Sangare, M. Mercier, G. Aubert, Solid State Commun. 52, 71 (1984).

[81] E. Kita, K. Siratori, K. Kohn, A. Tasaki, S. Kimura, I. Shindo, J. Phys. Soc. Jpn. 47, 1788 (1979). 
[82] T.M. Perekalina, I.S. Zheludev, E.M. Smirnovskaya, S.S. Fonton, N.A. Konoplev, Fiz. Tverd. Tela 16, 199 (1974).

[83] G.T. Rado, C. Vittoria, J.M. Ferrari, J.P. Remeika, Phys. Rev. Lett. 41, 1253 (1978).

[84] G.T. Rado, J.M. Ferrari, J.P. Remeika, J. Appl. Phys. 49, 1953 (1978).

[85] O.F. de Alcantara Bonfim, G.A. Gehring, Adv. Phys. (UK) 29, 731 (1980).

[86] J. Ohtani, K. Kohn, J. Phys. Soc. Jpn. 53, 3744 (1984).

[87] S. Foner, Phys. Rev. 130, 183 (1963).

[88] G.W. Pratt, P.T. Bailey, Phys. Rev. 131, 1923 (1963).

[89] E. Kita, K. Siratori, A. Tasaki, J. Phys. Soc. Jpn. 46, 1033 (1979).

[90] R. Fuchs, Philos. Mag. 11, 647 (1965).

[91] W. Kaiser, S. Sugano, D.L. Wood, Phys. Rev. Lett. 6, 605 (1961). 